\title{
Non-radially pulsating Be stars ${ }^{\star}$
}

\author{
Th. Rivinius ${ }^{1,2}$, D. Baade ${ }^{2}$, and S. Štefl ${ }^{3}$ \\ ${ }^{1}$ Landessternwarte Königstuhl, 69117 Heidelberg, Germany \\ 2 European Southern Observatory, Karl-Schwarzschild-Str. 2, 85748 Garching bei München, Germany \\ 3 Astronomical Institute, Academy of Sciences, 25165 Ondřejov, Czech Republic
}

Received 2 June 2003 / Accepted 19 August 2003

\begin{abstract}
Based on more than 3000 high-resolution echelle spectra of 27 early-type Be stars, taken over six years, it is shown that the short-term periodic line profile variability of these objects is due to non-radial pulsation. The appearance of the line profile variability depends mostly on the projected rotational velocity $v \sin i$ and thus, since all Be stars rotate rapidly, on the inclination $i$. The observed variability of the investigated stars is described, and for some of them line profile variability periods are given for the first time. For two of the investigated stars the line profile variability was successfully modeled as non-radial pulsation with $\ell=m=+2$ already in previous works. Since Be stars with similarly low $v \sin i$ share the same variability properties, these are in general explainable under the same model assumptions. The line profile variability of stars with higher $v \sin i$ is different from the one observed in low $v \sin i$ stars, but can be reproduced by the same model, if only the model inclination is modified to more equatorial values. Only for a few stars with periodic line profile variability the $\ell=m=2$ non-radial pulsation mode is not able to provide a satisfying explanation. These objects might pulsate in different modes (e.g. tesseral ones, $\ell \neq|m|)$. Almost all stars in the sample show traces of outburst-like variability, pointing to an ephemeral nature of the mass-loss phenomenon responsible for the formation of the circumstellar disk of early-type Be stars, rather than a steady star-to-disk mass transfer. In addition to the variability due to non-radial pulsation present in most stars, several objects were found to show other periods residing in the immediate circumstellar environment. The presence of these secondary periods is enhanced in the outburst phases. Short-lived aperiodic phenomena were clearly seen in two stars. But, given the unfavourable sampling of our database to follow rapid variability of transient nature, they might be more common. Only in two out of 27 stars short-term spectroscopic variability was not detected at all.
\end{abstract}

Key words. stars: emission-line, $\mathrm{Be}$ - stars: oscillations

\section{Introduction}

Classical Be stars are physically understood as rapidly rotating B stars with line emission arising from an ejected circumstellar disk in Keplerian rotation in the equatorial plane. In stars of early B subtype periodic line profile variability $(l p v)$ of the absorption is nearly omnipresent. The observed periods normally range from 0.5 to 2 days. Although in later-type Be stars such $l p v$ is seemingly absent (Baade 1989), lpv periods of this length are present in later B-type stars without emission lines, the so-called Slowly Pulsating B-stars (Waelkens et al. 1998,

Send offprint requests to: Th. Rivinius,

e-mail: T.Rivinius@lsw.uni-heidelberg.de

* Based on observations collected at the European Southern Observatory at La Silla, Chile, 55.D-0502, 56.D-0381, 58.D-0697, 62.H-0319, 63.H-0080, 64.H-0548, and 267.D-5702, the GermanSpanish Astronomical Centre, Calar Alto, operated by the MaxPlanck-Institute for Astronomy, Heidelberg, jointly with the Spanish National Commission for Astronomy, and on observations with the Wendelstein 80-cm and the Ondřejov 2-m telescopes, both equipped with the HEROs spectrograph provided by the Landessternwarte Heidelberg. and references therein). The variability of these SPB stars is generally accepted to be due to $g$-mode non-radial pulsation (nrp, see e.g. de Cat 2002).

Also some early-type non-emission line B stars have periods up to a few days. But upon more careful inspection their $l p v$ looks distinctly different than for the majority of Be stars. These non-Be stars are chemically peculiar objects with largescale magnetic fields, so that the period would be the one of the respective star's rotation, like in the cases of $\sigma$ Ori E, $\theta^{1}$ Ori C, or HD 105382 (e.g. Reiners et al. 2000; Briquet et al. 2001). Other stars of early B spectral subtype may have periods of less than twelve hours, and the lpv signature is typically that of radial pulsation or of non-radial $p$-modes, as in the $\beta$ Cephei type stars or in $\epsilon$ Per.

Although periodic variability is wide spread among all sorts of B stars, the presence of periodic slow lpv in early-type B stars and of circumstellar disks is strongly correlated. This could mean that the mechanism underlying the $l p v$ and the one leading to the Be phenomenon are causally related. Because, even after well over a century of observations, the latter is still not uncovered, it provides an additional incentive to carefully 
Table 1. The Be star observing campaigns.

\begin{tabular}{llllrrrr}
\hline \hline $\begin{array}{l}\text { Run } \\
\text { name }\end{array}$ & Season & Telescope & Instrument & $\begin{array}{r}\text { Run } \\
\text { length } \\
{[\mathrm{d}]}\end{array}$ & $\begin{array}{r}\text { Typ. \# of } \\
\text { spectra per } \\
\text { night \& object }\end{array}$ & $\begin{array}{r}\text { Resolving } \\
\text { power }\end{array}$ & $\left.\begin{array}{r}\text { Spectral } \\
\text { range } \\
{[\AA ̊}\end{array}\right]$
\end{tabular}

identify the nature of the $l p v$ of Be stars. This is complicated by the circumstance that the associated periods are equally well compatible with non-radial $g$-mode pulsation, rotation (since Be stars are rapid rotators, see Yudin 2001, and references therein), or even low Keplerian orbits above the photosphere.

In some single cases, fairly unambiguous evidence in support of the $n r p$ model for the Be star $l p v$ has been presented (Rivinius et al. 2001a: $\mu$ Cen; Maintz et al. 2003: $\omega \mathrm{CMa}$ ). However, although the models reproduce the observations in a stunning diversity of details, they only account for the strictly periodic part of the variability. While this usually is the dominant part, the unexplained residual variability would leave sufficient room for a hybrid explanation. In fact, several Be stars exhibit transient periods, which seem to be linked to the (unknown) mass transfer process from the central star to the rotating circumstellar disk. Some stars also show short-lived aperiodic variability, but for these no relation to the disk formation has been observed yet.

Studies of individual Be stars, like undertaken with HEROS and FEROs data of $\mu$ Cen and $\omega \mathrm{CMa}$, are immensely labor intensive. While such works will be required to answer the above questions in detail, a generalized picture of Be star lpv might be obtained best from a study of many objects, using a database as homogeneous as possible. Inspecting the Be stars in the large HERos database (e.g. Kaufer 1998, for an overview), some promise for such an approach lies in the impression that one of the parameters influencing the appearance of the $l p v$ the most is $v \sin i$. Since, in Be stars as rapid rotators, $v_{\text {rot }}$ covers a much smaller range than does $\sin i$ (the photospheric line broadening points to about 70 to $80 \%$ of the critical velocity, see Yudin 2001, and references therein), this would suggest that the viewing angle, $i$, has a large effect.

If so, there might be a standard model that can describe the periodic, the transient, or both variabilities in a fair fraction of all early-type Be stars. Such a model could, then, be studied further for possible implications for the explanation of the Be phenomenon. The first successful results from such a study of the uniformity of the periodic $l p v$, after correction for inclination angle, was published by Rivinius et al. (2002a). This paper now continues this work and mines the HEROS/FEROS database in full for both periodic and transient variability.

A brief overview of the observations leading to our database, which were described in detail already in previous publications, is given in Sect. 2. Section 3 then lists the $l p v$ properties of the individual stars studied, and Sect. 4 introduces a generalized $n r p$ model for the periodic $l p v$ of early-type Be stars, derived from the previous modeling of $\mu$ Cen and $\omega$ CMa. The applicability of this model and further consequences are discussed in Sect. 5, while Sect. 6 presents conclusions and outlook.

\section{Data and analysis method}

\subsection{Observational database}

Over the past eight years more than 3000 spectra of Be stars were obtained in the framework of the HEROs project and successors (Baade et al. 2002a). Except for a few objects selected because of their well known variability, like $\mu$ Cen and $\omega \mathrm{CMa}$, most targets were observed on account of their apparent brightness and celestial position. Shell stars are somewhat underrepresented in the data base. Since their photosphere is seen always through the disk an unambigiuous interpretation of observed variability is hampered in shell stars.

Most of the data were taken with the instruments HEROS ( $\approx 2300$ spectra, at various observatories) and FEROs ( $\approx 700$ spectra, at the ESO $1.5 \mathrm{~m}$ telescope). Both instruments are fiber-linked, predispersed echelle spectrographs, providing a large wavelength coverage over the Paschen continuum (and partly beyond) at high resolution (Table 1). The observations aimed at a $S / N$ of $100 \ldots 150$ with HEROS and $200 \ldots 300$ with FEROS, measured per extracted and rebinned pixel $(0.1 \AA$ wide for HEROS and $0.03 \AA$ for FEROS) in the stellar continuum close to $\mathrm{H} \beta$.

Details of HEROs were given by Kaufer (1998). The data reduction procedures are described in Rivinius et al. (2001b), 
Table 2. Observed stars. The spectral type and $v \sin i$ were either taken from Slettebak (1982) or from works dedicated to individual objects in detail, listed in Sect. 3. For brevity, only runs in which five or more spectra were obtained are listed for each star. Observed seasons correspond to the run designations in Table 1.

\begin{tabular}{|c|c|c|c|c|c|}
\hline Name & HR & HD & Sp. type & $\begin{array}{c}v \sin i \\
{\left[\mathrm{~km} \mathrm{~s}^{-1}\right]}\end{array}$ & $\begin{array}{l}\text { Observed seasons } \\
\text { (obtained spectra) }\end{array}$ \\
\hline$\alpha$ Eri & 472 & 10144 & B4 V(e) & 225 & 1s96MJ (5), 1s99a (20), 1s99b (42), 1s00 (44) \\
\hline DUEri & 1423 & 28497 & $\mathrm{~B} 1 \mathrm{Ve}$ & 230 & ls00 (6) \\
\hline DX Eri & 1508 & 30076 & $\mathrm{~B} 2 \mathrm{Ve}$ & 180 & ls00 (42) \\
\hline$\lambda$ Eri & 1679 & 33328 & B2 III(e)p & 250 & lsw97 (8), ca98 (29), 1s00 (44) \\
\hline$\omega$ Ori & 1934 & 37490 & B2 IVe & 160 & lsw97 (16), ca98 (20) \\
\hline $10 \mathrm{CMa}$ & 2492 & 48917 & B2 IIIe & 200 & ls00 (21) \\
\hline$\kappa \mathrm{CMa}$ & 2538 & 50013 & B2 IVe & 220 & $1 \mathrm{~s} 00(82)$ \\
\hline$\omega \mathrm{CMa}$ & 2749 & 56139 & $\mathrm{~B} 2.5 \mathrm{Ve}$ & 80 & ls96 (97), 1s97 (127), 1s99a (32), 1s00 (64), 1s02 (127) \\
\hline \multirow[t]{4}{*}{ FW CMa } & 2825 & 58343 & $\mathrm{~B} 3 \mathrm{Ve}$ & 40 & ls96 (9), ls97 (87), 1s00 (40) \\
\hline & 3642 & 78764 & B2 IVe & 120 & $1 \mathrm{~s} 99 \mathrm{~b}(6)$ \\
\hline & 4009 & 88661 & B2 IVe & 220 & ls $99 \mathrm{~b}(52), 1 \mathrm{~s} 00(66)$ \\
\hline & 4074 & 89890 & B3 III & 70 & ls $99 \mathrm{c}(15)$ \\
\hline PPCar & 4140 & 91465 & $\mathrm{~B} 4 \mathrm{Ve}$ & 250 & ls99b (55) \\
\hline \multirow[t]{2}{*}{$\delta$ Cen } & 4621 & 105435 & B2 IVe & 220 & $1 s 99 b(36)$ \\
\hline & 4625 & 105521 & B3 IVe & 130 & ls99b $(50)$ \\
\hline \multirow[t]{2}{*}{$\mu$ Cen } & 5193 & 120324 & B2 IV-Ve & 155 & 1s95 (97), 1s96 (87), 1s96MJ (115), ls97 (83), 1s99b (63), 1s99c (44) \\
\hline & 5223 & 120991 & B2 IVe & 70 & ls99c (8) \\
\hline$\eta$ Cen & 5440 & 127972 & B2 IVe & 350 & 1s95 (46), 1s96 (89), 1s96MJ (202), 1s97 (80), 1s99b (28), 1s99c (5) \\
\hline$\chi \mathrm{Oph}$ & 6118 & 148184 & $\mathrm{~B} 1.5 \mathrm{Ve}$ & 140 & ls99b (49) \\
\hline$\iota$ Ara & 6451 & 157042 & $\mathrm{~B} 2.5 \mathrm{IVe}$ & 320 & ls99b (38) \\
\hline$\alpha$ Ara & 6510 & 158427 & $\mathrm{~B} 3 \mathrm{Ve}$ & 250 & $1 s 99 b(43)$ \\
\hline $66 \mathrm{Oph}$ & 6712 & 164284 & B2 IV-Ve & 240 & ohp01 (5), ond (51) \\
\hline$\lambda$ Pav & 7074 & 173948 & B1.5 III & 170 & ls $99 b(11)$ \\
\hline 28 Cyg & 7708 & 191610 & B3 IVe & 320 & lsw97 (107), ca98 (107), wn00 (5), ond (88) \\
\hline $31 \mathrm{Peg}$ & 8520 & 212076 & $\mathrm{~B} 1.5 \mathrm{Ve}$ & 100 & ls99c (11), ohp01 (14), ond (18) \\
\hline$\pi$ Aqr & 8539 & 217571 & B1 III-IVe & 300 & ls99c (12), ohp01 (26), ond (11) \\
\hline$o$ And & 8762 & 217675 & B6 III & 260 & ca98 (104), wn00 (6), ond (110) \\
\hline
\end{tabular}

where also the complete $\mu$ Cen data is published. Feros, including the reduction, was introduced by Kaufer et al. (1997, 1999). Data from the observing runs at La Silla, both with HEROS and FEROs, were already introduced by Rivinius et al. (1998c) and Štefl et al. (2003a), assessing instrument stability and other questions.

The observations at La Silla were typically carried out in South American late summer with very good weather, so that the run lengths are almost equal to the number of nights with observations. For the European observations at the Landessternwarte Königstuhl, Calar Alto, and Wendelstein, only about 30 to $50 \%$ of the nights could actually be observed in. An idea of the sampling can be obtained by combining the information in Tables 1 and 2 for individual stars. Note that the typical sampling rates and $S / N$ ratios only exceptionally allowed to search also for higher modes with shorter periods.

Since August 2000, Heros is mounted at the $2 \mathrm{~m}$ telescope of the Czech Academy of Sciences in Ondřejov. As part of a long-term agreement, $50 \%$ of the total time is available to our group, typically in a two-week alternating rhythm.

A few dozen spectra were obtained 2001 by Y. Goranova, A. Bik, and M. Stuhlinger at Observatoire de Haute-Provence with AURÉLIE at the $1.52 \mathrm{~m}$ telescope during the 2nd NEON observing school.

\subsection{Period search- and modeling-techniques}

Be stars are known to undergo long-term changes due to the varying amount of circumstellar matter, potentially affecting the results of period determinations. Echelle spectra, however, offer the advantage to investigate a large number of lines. At least some lines can always be found which are known to be hardly formed in the disk, and thus should be only little affected by these long-term changes. In early type Be stars these are lines of ions like Si III, or higher-term He I lines, e.g. those at 4026 or at $4388 \AA$. Though it cannot be entirely exluded that part of the spectra are affected by transiently present periods (in particular for those objects observed only in a single season), for most targets the results are ascertained by multi-seasonal observations and the limitation to uncontaminated lines (see above).

In these lines, first the mode (position of the line center) was measured automatically and a period search using a Fourier technique based on Scargle's (1982) algorithm performed. The results were checked against a period search on the full line profile, i.e. applying the above Fourier technique to the local intensities across the line width. In objects where multiple periods were found, these were iteratively removed using the procedure described by Kaufer et al. (1996). This procedure is fitting a sine-wave to the data, and repeating the 
Table 3. Be stars in the sample for which spectroscopic periods could be established. The error of these periods in frequency space can be estimated from the overall observing length in Table 1. The photometric periods are taken from various sources discussed in detail in Sect. 3. For photometric periods that agree with the main spectroscopic period (or one of its aliases) the amplitudes are typeset in italic. The spectroscopic lpv is of $\omega$ CMa type (see Sect. 5.1) in all listed stars, except for $\lambda$ Eri, $\kappa \mathrm{CMa}$, and $o$ And.

\begin{tabular}{|c|c|c|c|c|}
\hline \multirow[t]{2}{*}{ Name } & \multirow[t]{2}{*}{ HR } & \multicolumn{2}{|r|}{ Periods } & \multirow{2}{*}{$\begin{array}{c}\text { Photom. } \\
\text { amplit. } \\
\text { [mag] }\end{array}$} \\
\hline & & $\begin{array}{l}\text { Spectr. } \\
\text { [d] }\end{array}$ & $\begin{array}{c}\text { Photom. } \\
\text { [d] }\end{array}$ & \\
\hline$\alpha$ Eri & 472 & 1.291 & $1.26^{1}$ & 0.020 \\
\hline DUEri & 1423 & 0.577 : & $0.654^{2}$ & 0.020 \\
\hline DXEri & 1508 & 1.267 & $1.267^{3}$ & 0.015: \\
\hline$\lambda$ Eri & 1679 & 0.702 & $0.702^{4}$ & 0.010 \\
\hline $10 \mathrm{CMa}$ & 2492 & 1.338 & $1.338^{2}$ & $0.007:$ \\
\hline$\kappa \mathrm{CMa}$ & 2538 & 0.548 & $1.337^{2}, 1.408^{5}$ & 0.015: \\
\hline$\omega \mathrm{CMa}$ & 2749 & 1.372 & $1.47,1.37^{6}$ & $<0.005$ \\
\hline \multirow[t]{4}{*}{ FW CMa } & 2825 & 0.839 & - & - \\
\hline & 3642 & 1.130 & - & - \\
\hline & 4009 & 0.650: & - & - \\
\hline & 4074 & 2.318 & - & - \\
\hline PPCar & 4140 & $0.504:$ & - & - \\
\hline \multirow[t]{2}{*}{$\delta$ Cen } & 4621 & 1.139: & $1.923^{4}, 0.55,1.1^{7}$ & 0.010 \\
\hline & 4625 & 1.686 & - & - \\
\hline$\mu$ Cen & 5193 & 0.503 & $1.06^{8}$ & $<0.005$ \\
\hline$\eta$ Cen & 5440 & 0.577 & $0.64^{9}$ & 0.030 \\
\hline$\chi \mathrm{Oph}$ & 6118 & 0.649 : & $>0.45^{2}$ & 0.025 \\
\hline 66 Oph & 6712 & 0.452 & - & - \\
\hline $28 \mathrm{Cyg}$ & 7708 & 0.648 & $0.64^{10}$ & 0.025 \\
\hline 31 Peg & 8520 & 0.724: & - & - \\
\hline$o$ And & 8762 & 0.694 & $1.58,0.79,0.69^{11}$ & 0.019 \\
\hline
\end{tabular}

References: ${ }^{1}$ Balona et al. (1987), ${ }^{2}$ Hubert \& Floquet (1998), ${ }^{3}$ Štefl \& Balona (1996), ${ }^{4}$ Balona et al. (1992), ${ }^{5}$ Balona (1990), ${ }^{6}$ Štefl et al. (1999), ${ }^{7}$ Percy et al. (2002), ${ }^{8}$ Cuypers et al. (1989), ${ }^{9}$ Śtefl et al. (1995), ${ }^{10}$ Balona (1995), ${ }^{11}$ Sareyan et al. (1998).

Fourier-transformation on the residuals to find the next period (which is mathematically equivalent to a CLEAN-type algorithm with gain unity). Besides returning a consistent period by both techniques (across the full profile), a coherent phase propagation was required for acceptance as significant period (for instance, see Figs. 1 and 7 of Rivinius et al. 1998b, also describing the period search method in detail).

The line profile variations associated to the detected periods are then compared to a pulsational model, based on Townsend's code (1997). This model was shown by Maintz et al. (2003) to explain the lpv of the prototypical star $\omega \mathrm{CMa}$ very well, namely with a monoperiodic retrograde sectorial pulsation mode with indices $\ell=m=+2$. More detailed properties of this model are given in Sect. 4 and by Maintz et al. (2003).

\section{Individual objects}

In the following we summarize findings about the observed stars, both derived from own data and the literature. For most of the stars in Table 2 sufficient data were obtained to study the periodic line profile variability. For many the periodic behavior was found in previous studies already. But wherever possible the period analyses were re-performed on the HEROs and FEROs data, using the published periods as initial guesses (Table 3).

Figures 1 to 5 show the phase-sorted residual lpv of stars with $\omega$ CMa like behavior (see Maintz et al. 2003, see also Sect. 5.1), in order of increasing $v \sin i$. In Fig. 6, the three stars with different variability are presented. Each star is briefly commented in the following:

$\alpha$ Eri: Balona et al. (1987) derived a period of 1.26 days from simultaneous spectroscopy and photometry. Later, Porri \& Stalio (1988) showed spectra displaying asymmetric $l p v$. The period derived here, $1.291 \mathrm{~d}$, bases on observations taken from 1996 to 2000 . The seasons have been analyzed both individually and as combined data, all pointing to the longer period as used here.

Due to its brightness, $\alpha$ Eri became a frequently observed target also with space experiments. Smith (2001), analyzing the UV spectrophotometric variability observed with IUE, concluded the stellar continuum to vary with higher amplitude in the blue part than in the red, but not showing distinct spectral features that would clearly point to either $n r p$ or other investigated scenarios causing the variations.

The recent measurement of $\alpha$ Eri's large equatorial radius of $12 R_{\odot}$ and high rotational flattening, together with the rotational velocity of at least $225 \mathrm{~km} \mathrm{~s}^{-1}$ exclude the $l p v$ period to be rotational (Domiciano de Souza et al. 2003).

DU Eri: For this star only very few spectra are available, so that these are sorted only into 8 phase bins instead of 16 (Fig. 3), as with the other figures. The period used to sort the data is very tentative with $0.577 \mathrm{~d}$. However, the variable shape of the individual line profiles clearly is of the same type of variability as for the other pulsating stars in this sample, i.e. the symmetry of the profile changes in a low-order fashion. Dachs \& Lemmer (1991) found a double-wave period of $0.65 \mathrm{~d}$, while from HIPPARCOS photometry a single-wave value of $0.654 \mathrm{~d}$ was derived (Hubert $\&$ Floquet 1998). Smith (2001) found DU Eri to exhibit $n r p$ type spectrophotometric UV variability with a period of $1.2 \mathrm{~d}$, which is about twice the period presented here, while Peters \& Gies (2000) found a period of $0.61 \pm 0.08 \mathrm{~d}$ in the UV line profiles and $1.2 \mathrm{~d}$ in the UV wind lines, tentatively proposing an $\ell=-m=2$ mode.

DX Eri: Štefl \& Balona (1996) investigated this star in detail, deriving a consistent period from both photometry and spectroscopy of $1.26708 \mathrm{~d}$. This period is confirmed by FEROS observations in 2000. The circumstellar emission increased somewhat during the two weeks of observations, but there was no obvious outburst. The period derived from Hipparcos photometry, 1.383 d (Hubert \& Floquet 1998), does not sort the data well and might be an alias, but could also indicate transient activity, as described in Sect. 5.5.

$\lambda$ Eri: The $l p v$ period was derived in many independent studies to be $0.702 \mathrm{~d}$. Kambe et al. $(1993,1998)$ carried out an extensive campaign over several seasons and found not only this period, but a second coherent one of $0.269 \mathrm{~d}$ and intermittently present ones of $0.6 \mathrm{~d}$ and $0.75 \mathrm{~d}$. 

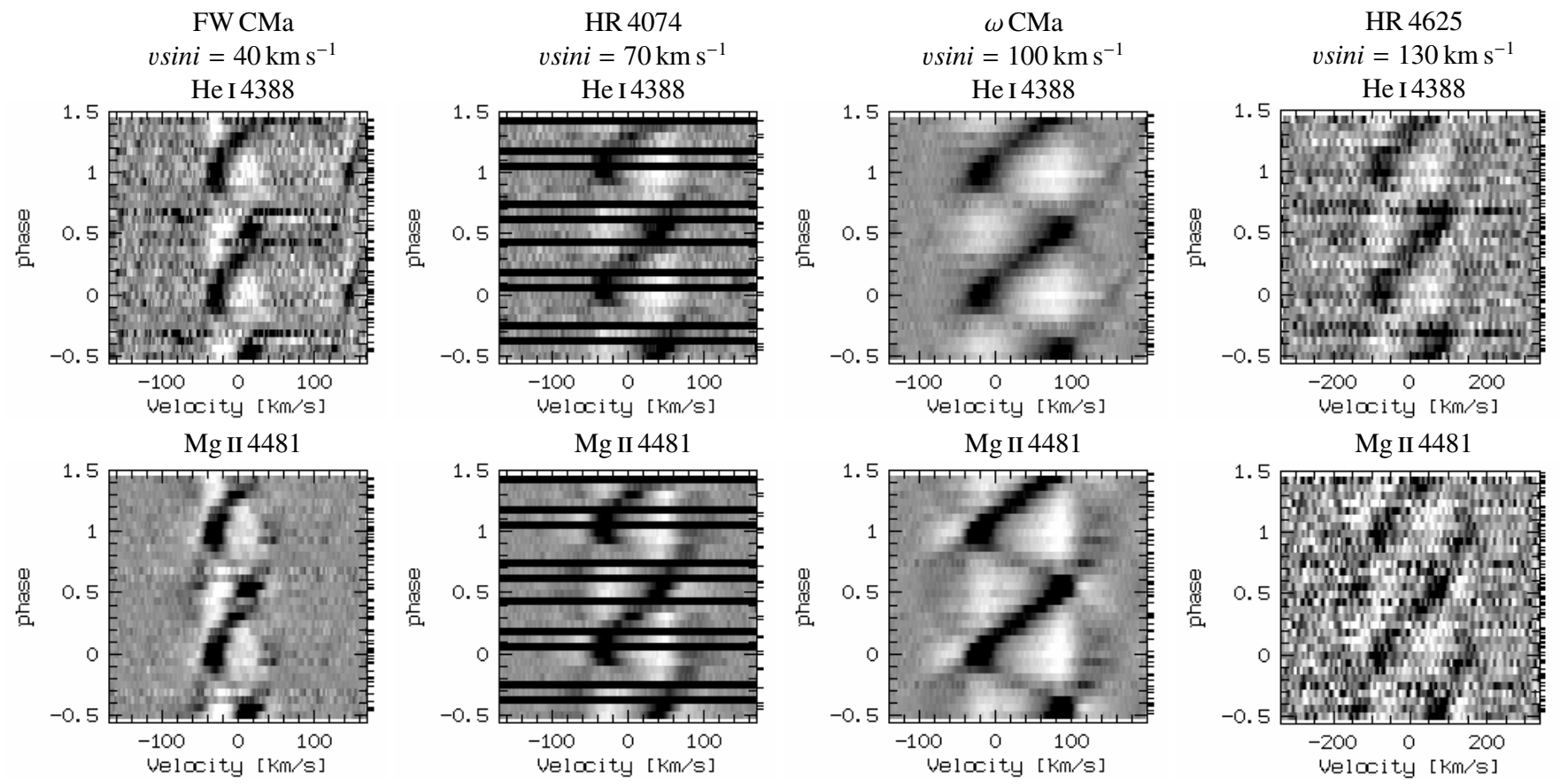

Fig. 1. Phased main $l p v$ in He I 4388 and Mg II 4481 of $\omega$ CMa like Be stars with low $v \sin i$. Note the typical variability of the far wings (beyond $v \sin i$ ) in antiphase to the core, seen better in metal lines like Mg I 4481 than in He I lines. The periods are given in Table 3.
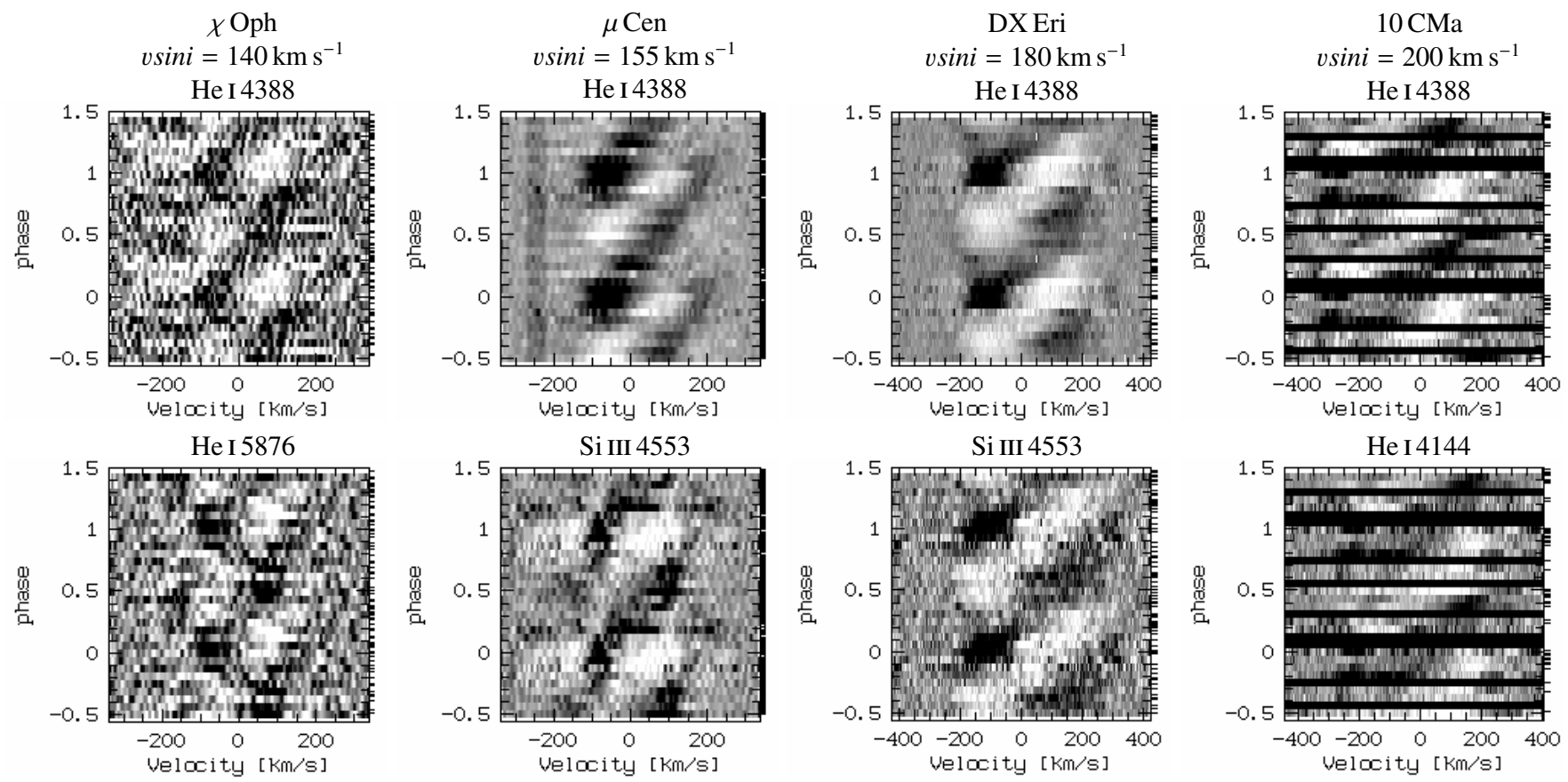

Fig. 2. Phased main $l p v$ of $\omega$ CMa like Be stars with intermediate $v \sin i$ in He I 4388 and selected metal lines, in which the variability is well seen. In stars with variability too weak to be detected in metal lines, a second He I line is shown. The periods are given in Table 3.

The appearance of those intermittent periods is similar to the transient ones found in $\omega \mathrm{CMa}, \mu$ Cen and $\eta$ Cen by Štefl et al. (1998). $\lambda$ Eri is one of the few stars with clearly different $l p v$ than $\omega \mathrm{CMa}$ (Fig. 6). Although the general appearance is that of low-order variation, the $l p v$ shows additional substructure in particular in the line wings.

Smith (1989) reported a large number of aperiodic smallscale transient features. Such features are weakly seen also in FEROS data, but are not detected in the noisier (and less resolved) HERos data. The sampling, optimized for phase coverage of the $0.7 \mathrm{~d}$ period, does not trace those features, and is therefore not suited for their investigation.

$\omega$ Ori: Although the data is not sufficient to cover the unfavorable period of almost exactly one day, the type of $l p v$ as shown by Neiner et al. (2002, their Fig. 16), similar to most other Be stars, can be confirmed.

10 CMa: Baade (1984b) found periodic $l p v$ and gave a period of $1.36 \pm 0.05 \mathrm{~d}$. The 21 FEROS spectra taken 2000 confirm 

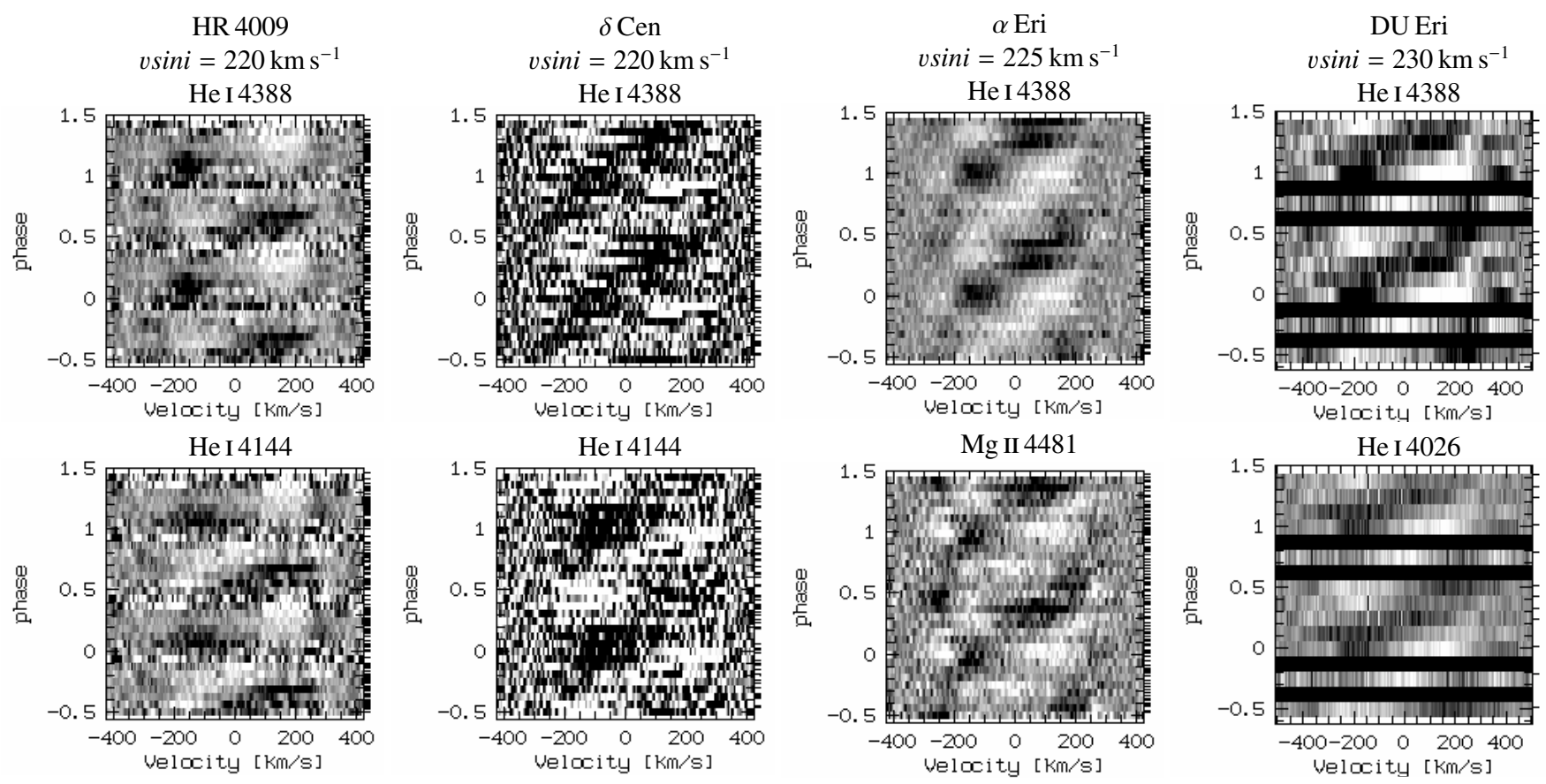

Fig. 3. Phased main $l p v$ of $\omega \mathrm{CMa}$ like Be stars with intermediate to high $v \sin i$. The periods are given in Table 3 .
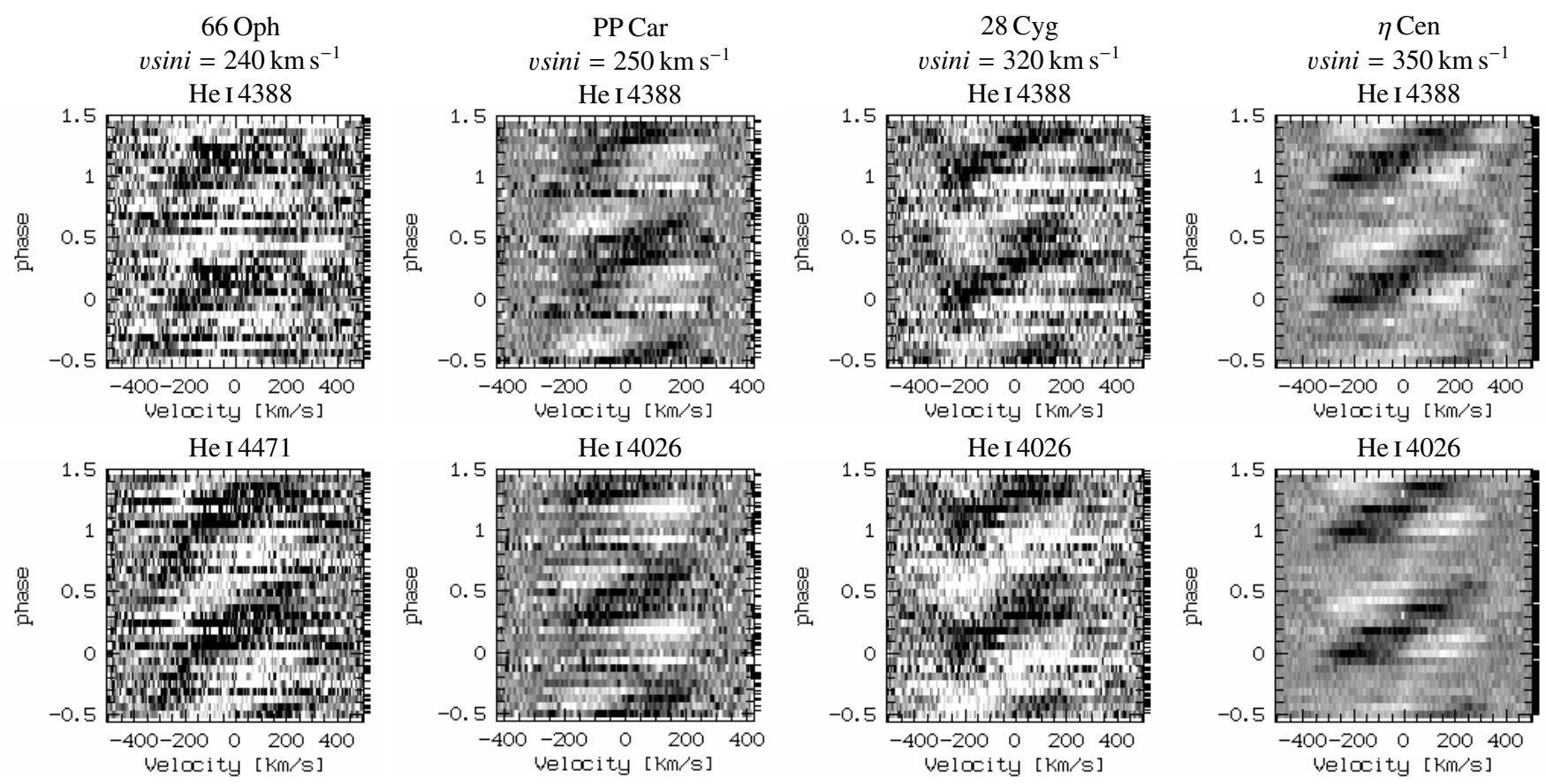

Fig. 4. Phased main $l p v$ of $\omega$ CMa like Be stars with high $v \sin i$. The periods are given in Table 3 .

this value, although the latter spectra alone do not suffice to distinguish between this period and its 1-day aliases. Dachs \& Lemmer (1991) found a photometric $1.34 \mathrm{~d}$ period, later Balona et al. (1992) reported a double wave period of $2.632 \mathrm{~d}$, which is just about twice these values. HIPPARCos photometry gives $1.338 \mathrm{~d}$ (Hubert \& Floquet 1998), but Percy et al. (2002) did not confirm this value and noted a period of $0.5 \mathrm{~d}$ to be more consistent with the data. $\kappa$ CMa: This star was observed intensively with FEROs in 2000. The period that sorts the $l p v$ best is $0.548 \mathrm{~d}$ with no confusion due to aliasing. The residual $l p v$ is one of the few that does not resemble the $\ell=m=2$ patterns derived from modeling (Sect. 4). Instead of a single low-order bump traveling from blue to red in the line profile, a similar bump is present, but shows clear substructure in metal lines, as if two such patterns would follow each other with a separation of about 0.25 cycles (Fig. 6). 

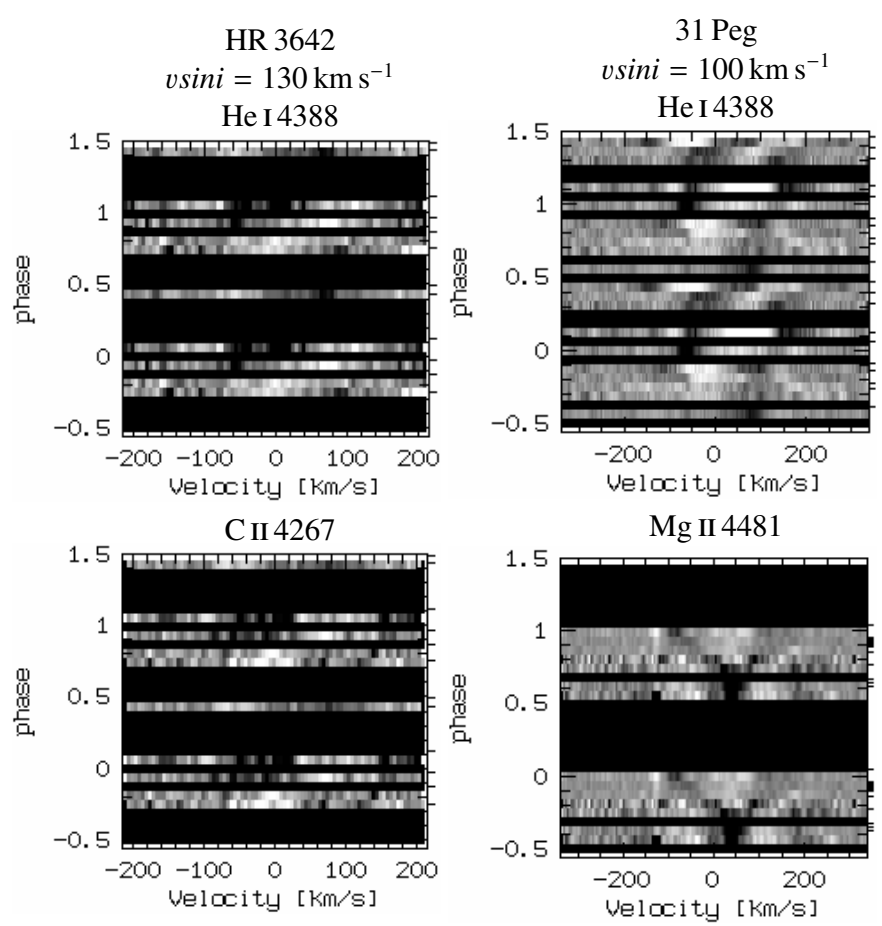

Fig. 5. Phased main $l p v$ of HR 3642 and $31 \mathrm{Peg}$. The period for HR 3642 was taken from Carrier et al. (2002). For 31 Peg the 1s99c data is shown for He I 4388, the ohp01 data for Mg II 4481 (see Table 1 for notation used for observing runs). The periods are given in Table 3.

$\kappa$ CMa shows also a secondary period of $0.617 \mathrm{~d}$ with the typical properties of what Štefl et al. (2003a) called a transient period in $\omega \mathrm{CMa}$. Only sparse data were taken in other observing seasons $(1997,1999,2002)$, but already these few data make clear that $\kappa \mathrm{CMa}$ is extraordinarily active on short timescales. Comparison with the outbursts directly witnessed in $\mu$ Cen and $\omega$ CMa suggests that $\kappa \mathrm{CMa}$ underwent such outbursts most of the time when it was observed. Photometric period determinations do not agree with these spectroscopic values. Percy et al. (2002) confirm the short periodic behavior found by Hubert \& Floquet (1998, $1.337 \mathrm{~d}$ ), while Balona (1990) derived $1.408 \mathrm{~d}$ from 1988 data, but data obtained 1987 just previous to a photometric outburst seem to be sorted better with $\approx 1 \mathrm{~d}$ (cf. his Fig. 5).

$\omega$ CMa: The periodic $l p v$ of this object has been discussed in detail already by Štefl et al. (2003b) and modeled by Maintz et al. (2003, see also Sect. 4). The outbursts of $\omega$ CMa are described in Štefl et al. (2003a). During such outbursts, a secondary period of about $1.47 \mathrm{~d}$ is apparent. It is seen only in lines with at least some circumstellar contribution, but not in purely photospheric lines. There is only very weak photometric variability, which is not dominated by the photospheric $1.37 \mathrm{~d}$ period, but the only transiently present $1.49 \mathrm{~d}$ period. For a detailed discussion of the variability and previous investigations the publications listed above are referred to.

FW CMa: The period of $0.83 \mathrm{~d}$ has tentatively been published already on account of the first HEROs data (Rivinius 1999). Further observations confirmed this period as coherent at least between 1996 and 2000. The star is not known to show periodic behavior in photometry, but already Hanuschik et al. (1996) found FW CMa comparable to $\omega \mathrm{CMa}$ in terms of the emission outburst behavior. This was confirmed with HEROS and FEROS spectra.

HR 3642: Carrier et al. (2002) detected short-periodic $l p v$ with $1.13028 \mathrm{~d}$ in spectroscopic data, while no such period was present in HIPPARCos photometry. The variability shown (their Figs. 13 to 15) looks like the $l p v$ presented here of objects of similarly low $v \sin i$. $l p v$ is also clearly present in our few spectra of this star, but only six spectra at a relatively low $S / N$ of 60 do not allow to search for this period independently or determine the type of the $l p v$. Adopting the given period, however, the data is acceptably sorted (Fig. 5).

HR 4009: No period was given for this star so far. A periodic signal in the analysis is very weak, but undoubtedly present in both observing seasons in 1999 and 2000. Next to the periodic lpv HR 4009 shows also short-lived aperiodic phenomena (Fig. 7), which resemble the ones reported by Smith (1989) for $\lambda$ Eri.

HR 4074: The spectroscopic $l p v$ found initially by Baade (1984b) is very similar to the one of $\omega \mathrm{CMa}$. The star was classified as a Be star from a spectrum taken in the 19th century, but currently does not show any traces of circumstellar material (Štefl et al. 2002, and references therein), excluding any interpretation of its variability in terms of circumstellar matter. Percy et al. (2002) do not find shortperiodic variability in HIPPARCos photometry.

PP Car: The period analysis performed here indicates a weak signal, but clearly above the noise at about $1.0 \mathrm{~d}$ or its $1-\mathrm{d}$ aliases. Porri \& Stalio (1988) published spectra in which $l p v$ is apparent, and a period of $0.35 \mathrm{~d}$ is suggested for the variability of the full width at half maxima of the He I 6678 line. In fact, the extreme red asymmetry of the profiles in their data (their Fig. 8) repeats on a timescale of slightly more than a day. In one case, the opposite asymmetry was observed $0.22 \mathrm{~d}$ earlier. Computing the phase differences resulting from the potential period values $(1.0,0.504$, and $0.33 \mathrm{~d}$ ), phase differences of $0.21,0.44$ and 0.66 cycles are derived. Similar to the arguments for $\mu$ Cen (Rivinius et al. 1998 b), a phase difference of 0.44 cycles between these two spectra (i.e. close to 0.5 for opposite asymmetry) seems more likely than the others, so we adopt $0.504 \mathrm{~d}$ as $l p v$ period, but still the one day aliases cannot firmly be excluded with the current data.

$\delta$ Cen: Balona et al. (1992) report a tentative photometric period of $1.923 \mathrm{~d}$ seen in 1988, but absent in 1990. Percy et al. (2002) find either 0.55 or $1.1 \mathrm{~d}$ in HIPPARCos photometry, favoring the shorter value. In the 1999 HEROs spectroscopy, also both values are present, $0.532 \mathrm{~d}$ and $1.139 \mathrm{~d}$, being 1-d aliases of each other, but with a formally somewhat higher significance of the longer period, which is adopted here.

HR 4625: Unpublished single previous spectra taken by S. Stefl suggested this star to be a good candidate to search for periodic $l p v$. It was observed intensively with HEROS in 1999. Over the $2 \frac{1}{2}$ months of observations no outburst occurred, but the Balmer emission underwent the 

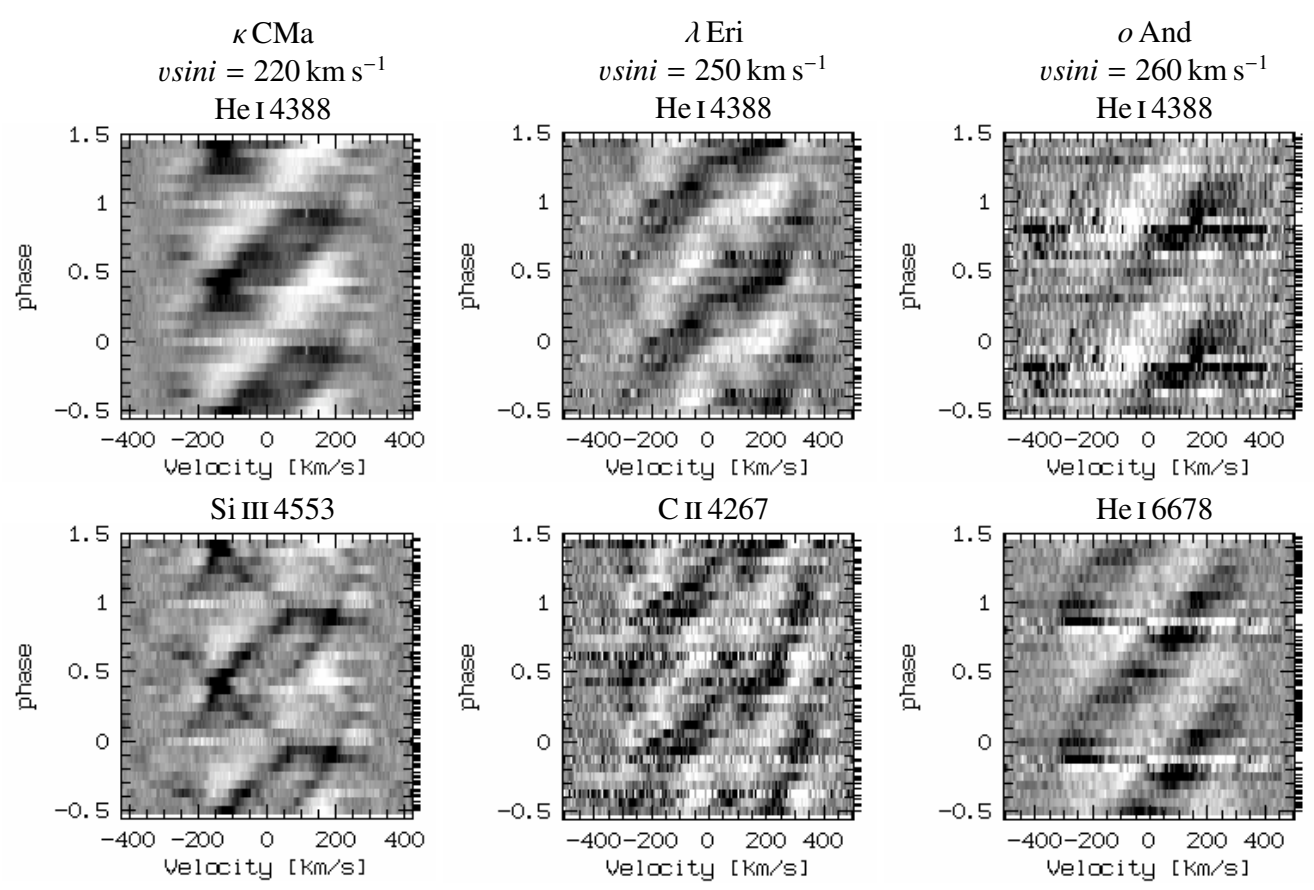

Fig. 6. Phased $l p v$ of the Be stars which do not show $\omega$ CMa-like behavior.

CII 4267

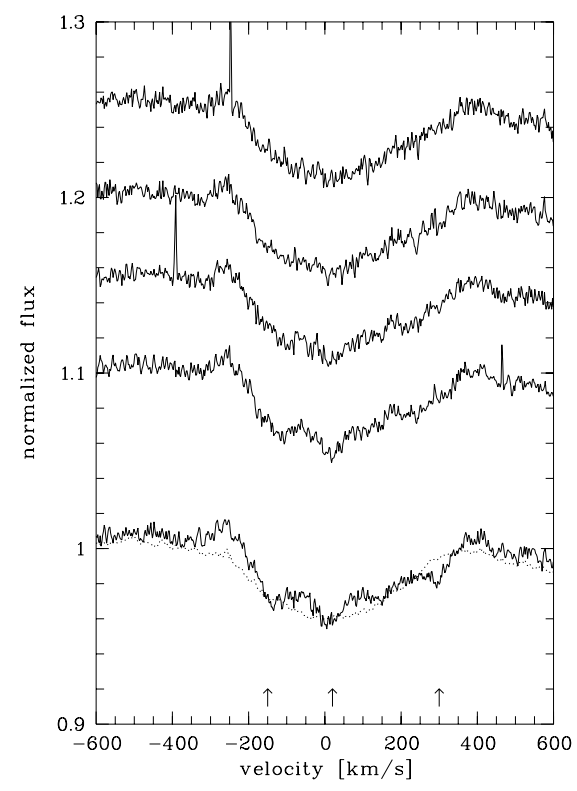

HeI 4471

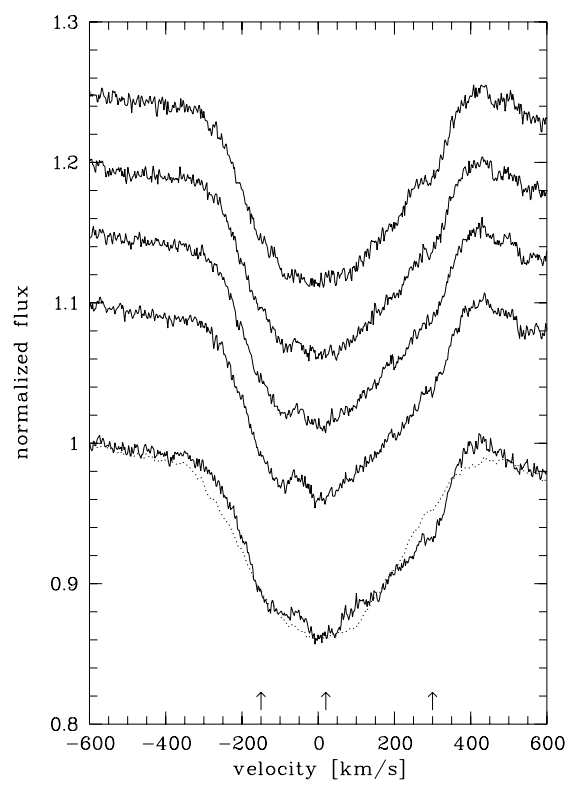

HeI 6678

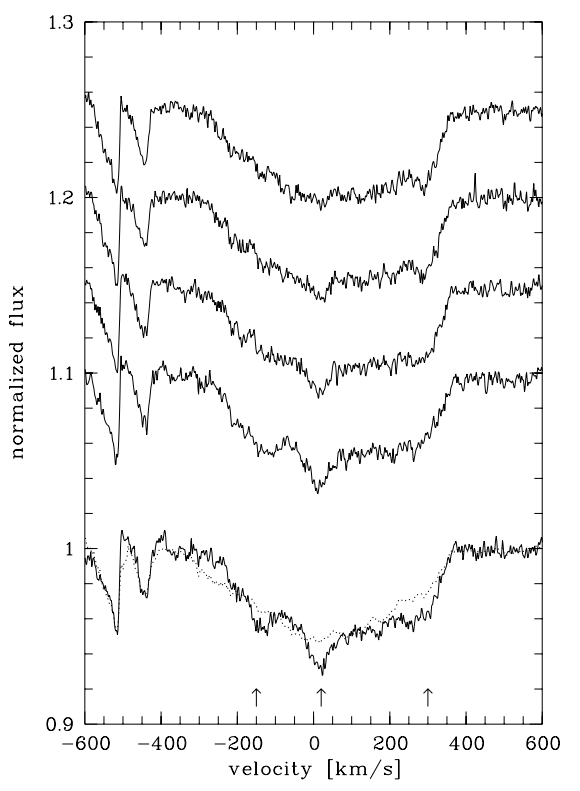

Fig. 7. Evolution of three short-lived transients in three different lines of HR 4009 observed with FEROs. The dotted line overplotted to the lowermost profiles corresponds to the mean of all observations. The structure between -600 and $-400 \mathrm{~km} \mathrm{~s}^{-1}$ in He I 6678 is due to detector blemishes. Time increases towards the top, proportional to the offset. The observations span 95 min.

typical evolution of a relaxation phase, i.e. the wings became narrower while the emission height increased. The photospheric lines showed typical $l p v$ with a period of $1.69 \mathrm{~d}$.

$\boldsymbol{\mu}$ Cen: This multiperiodic Be star was investigated in detail by Rivinius et al. (1998b,c). Modeling the short-periodic $l p v$ resulted in the same mode properties as for $\omega \mathrm{CMa}$ (Rivinius et al. 2001a). The spectral data was made public by Rivinius et al. (2001b). Only the main period of
0.503 day is shown here. In addition to this period there are three more periods with very similar values, together called the "longer period group" by Rivinius et al. (1998b), and two more periods at $0.28 \mathrm{~d}$ which constitute the "shorter period group". Similar to $\omega \mathrm{CMa}$, there are also transient periods present during outbursts.

From several independent photometric data sets a period of about $1.06 \mathrm{~d}$ was derived (Cuypers et al. 1989; Dachs \& Lemmer 1991; Percy et al. 2002). For this reason 


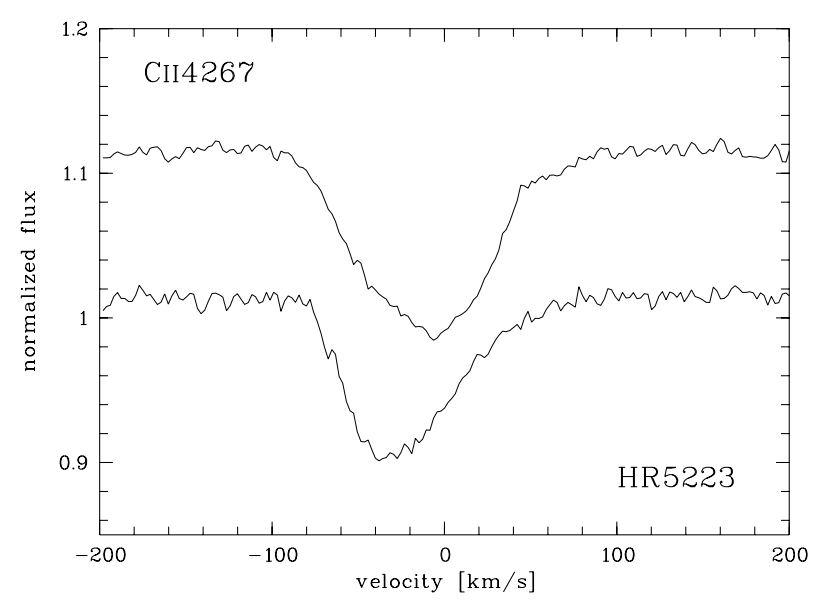

Fig. 8. The $l p v$ of HR 5223. The few spectra do not enable a period determination, but the $l p v$ is of the same type as in FW CMa, which has a similarly low $v \sin i$. The spectra are the two most different ones observed.

Balona et al. (2001b) favor a 1d double-wave period over a $0.5 \mathrm{~d}$ single wave also for spectroscopy. However, neither is the photometric period a double wave, nor do the two assumed half-waves for spectroscopy differ from each other, which in the absence of other evidence, is the only justification to assume a double-wave pattern. The secondary period group at $0.28 \mathrm{~d}$, also due to non-radial pulsation, but in a higher mode, is not seen in photometric data at all.

HR 5223: During the 20 nights spanned by the observations, the broad Balmer emission wings decreased, while the $\mathrm{H} \alpha$ peak height rose. This is a usual early post-outburst evolution, observed in detail e.g. in $\omega \mathrm{CMa}$, FW CMa, and $\mu \mathrm{Cen}$. Low-order $l p v$ is clearly present in the 8 observed spectra. The two spectra with the most extreme asymmetry (Fig. 8) compare well with the typical appearance of asymmetric phases in other low $v \sin i$ stars. No period can be derived from the limited data, however.

$\eta$ Cen: The photometric period given by Cuypers et al. (1989) is $1.927 \mathrm{~d}$ with a triple-wave shape. Štefl et al. (1995) took into account both photometric and spectroscopic data and derived a single-wave period of $\frac{1}{3}$ of the triple-wave value, $0.642 \mathrm{~d}$, which was confirmed by later works (e.g. Janot-Pacheco et al. 1999; Balona 1999; Levenhagen et al. 2003). Štefl et al. (1995) also detected traces of a period at about $0.57 \mathrm{~d}$, but due to unfortunate sampling could not confirm its reality. Such a shorter period was, however, also found by Janot-Pacheco et al. (1999) and Levenhagen et al. (2003), in the HERos data, and by Balona (1999). The latter author rejected this period as not real, since it was not found in all spectroscopic lines, but this is similar with the He I vs. Si III lines of e.g. $\mu$ Cen (Rivinius et al. 1998b). Given all the detections in independent data, however, $\eta$ Cen can be concluded to be a multiperiodic Be star. Taking into account the obtained results about secondary "transient" periods in other stars, the photospheric main period should be the shorter one, which is weaker in photometry, but almost dominant in He I lines.
Analysis of the data of all seasons from 1995 to 1999 permits the $0.57 \mathrm{~d}$ period to be resolved into two with values of $0.577 \mathrm{~d}$ and $0.565 \mathrm{~d}$. Although the weaker one is also weaker than the $0.642 \mathrm{~d}$ secondary period, it is clearly detected in all stronger He I lines.

Sorting the data with the shorter periods, the patterns are well comparable to other Be stars (Fig. 4). In fact, both 0.577 and $0.565 \mathrm{~d}$ have very similar patterns, just like the group of longer periods present in $\mu$ Cen (Rivinius et al. 1998b). Similar to other high $v \sin i$ Be stars, these periods are only very weakly present in metal lines like Si III, but still detectable in e.g. C II 4267.

For the secondary period, i.e. $0.642 \mathrm{~d}$, which was the only one taken into account by most previous investigators, striking differences are seen between the $l p v$ of $\mathrm{H} \delta$, He I 6678, and He I 4388 lines (Fig. 15, discussed in Sect. 5). Also this secondary period is coherently present through all observing seasons from 1995 to 1999 . The same variability pattern can be identified in Fig. 10 of Balona (1999), but since the main period of $0.577 \mathrm{~d}$ and eventual transient features will not perfectly cancel out during such a short run of twenty days, the pattern is much less evident. $\eta$ Cen shows line emission outbursts about once every couple of weeks. This is almost as frequent as in $\mu \mathrm{Cen}$, but the outbursts of $\eta$ Cen are less prominent, and detectable only in data with relatively good spectral coverage and $S / N$. The beat frequency of the two main periods $(0.565 \mathrm{~d}$ and $0.577 \mathrm{~d}$ ) is in the same order of magnitude, $29 \mathrm{~d}$, but the outbursts are not regular enough to be explained with just these two frequencies. The secondary period, $0.642 \mathrm{~d}$, is unlikely to contribute to such a beating mechanism, as it probably is not associated with photospheric $n r p$.

$\chi$ Oph: Hubert \& Floquet (1998) reported cyclic behavior of the HIPPARCos photometry, but could only constrain the period to be $>0.45 \mathrm{~d}$. The spectroscopic HERos data also shows a periodic signal, but cannot distinguish between $0.649 \mathrm{~d}$ and the 1 -day alias at $0.393 \mathrm{~d}$. Re-investigating HIPPARCos data Percy et al. (2002) propose 0.55 d. Taking into account the results by Hubert \& Floquet (1998), $0.649 \mathrm{~d}$ is adopted for this work.

$\iota$ Ara: There is no $l p v$ apparent for the available HERos data. A period of $\approx 0.56 \mathrm{~d}$ was derived by Cuypers et al. (1989), switching from single- to double-wave appearance, while Dachs \& Lemmer (1991) published a 0.61 d double-wave period.

From HIPPARCos data, Hubert \& Floquet (1998) deduced $0.565 \mathrm{~d}$, not mentioning a double wave nature. If this period is present also in spectroscopy, it is below the detection threshold for HERos data, which shows the spectrum to be stable within the limits. The star has not been observed with FEROS, which would have provided better resolution at higher $S / N$.

$\boldsymbol{\alpha}$ Ara: Neither the photometric period reported by Cuypers et al. (1989), $0.9807 \mathrm{~d}$ with a double-wave lightcurve, nor the single wave period of $0.490 \mathrm{~d}$ found by Hubert \& Floquet (1998) can be confirmed by spectroscopy. Although these periods are close to one day or half a day, the phase coverage would almost be complete for both 


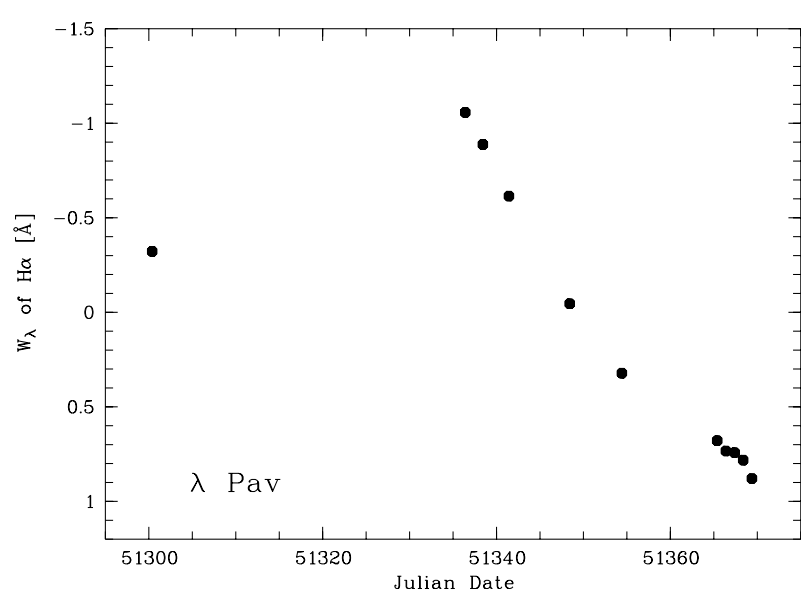

Fig. 9. The $\mathrm{H} \alpha$ equivalent width of $\lambda$ Pav. An outburst probably occurred between JD 51300 and 51 340, confirming the photometric outburst behavior of $\lambda$ Pav found by Hubert \& Floquet (1998).

of them in our data, so this is not a sampling problem. However, a slow secular shift in the overall velocity of the emission lines is apparent, so that $\alpha$ Ara could be a binary with a period longer than the run length of $71 \mathrm{~d}$. This would severely hamper the detection of short periods in data with one spectrum per night.

66 Oph: A spectroscopic period of $0.45 \mathrm{~d}$, together with a shorter one of $0.25 \mathrm{~d}$, was published recently by Floquet et al. (2002) and ascribed to nrp. While the data available for the present work are not suited to confirm the shorter period, the $0.452 \mathrm{~d}$ sort the HEROs spectra well.

Štefl et al. (2003c) report 66 Oph to be a spectroscopic triple system similar to $o$ And, where a close pair of late B/early A type stars is seen in singly ionized metal lines. This pair orbits the Be star on a very long timescale at large distance, so that no influence on the Be star is to be expected.

$\lambda$ Pav: In the spectra $l p v$ is apparent, but the limited database of 11 spectra over 70 days does not allow one to determine a period. The variability is close to the detection threshold, so that the individual profiles neither permit the variability type to be derived from them, like in HR 5223. Hubert \& Floquet (1998) give a photometric period of $0.6 \mathrm{~d}$ and note outburst behavior. The Balmer emission during our observations was highly variable (Fig. 9), comparable to $\mu$ Cen in its "flickering" stage (Hanuschik et al. 1993).

28 Cyg: Periodic behavior with $0.7 \mathrm{~d}$ was initially found by Spear et al. (1981) in photometry and later confirmed as $0.68 \mathrm{~d}$ by Peters \& Penrod (1988). Further works agree with this period, and also found other periods, which were not present in all data sets, however (Pavlovski et al. 1997, for a review). The period given here, $0.64 \mathrm{~d}$ was found also by Hahula \& Gies (1994). The values ranging from $0.64 \mathrm{~d}$ to $0.7 \mathrm{~d}$ seem incompatible. But the multiperiodicity with two close periods around $0.64 \mathrm{~d}$ reported by Tubbesing et al. (2000), based on a subset of the HEROs data presented here, potentially explains slightly different periods found from run to run, if these runs are too short to resolve the period doublet.

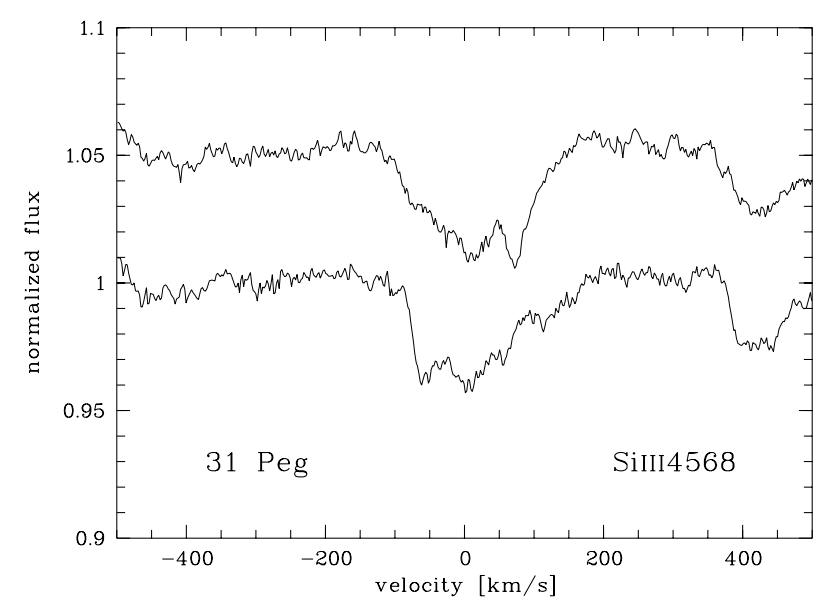

Fig. 10. $\omega$ CMa-like spikes in the lpv of 31 Peg observed with FEROS.

31 Peg: The period tentatively given here, $0.724 \mathrm{~d}$, was derived from the 1s99c and ohp01 data independently, but both data sets suffer from 1-d aliasing. No other period determination was published by now. The presence of rapid $l p v$ itself is, however, clearly seen in the ohp01 data, which consists of 14 spectra taken in two separate nights.

The $l p v$ is also obvious in the 1s99c data and published data (e.g. Zorec et al. 1996), and several spectra clearly show spikes like in $\omega$ CMa (Fig. 10). Metal line emission was strong in 1999, but weak in the ohp01 and ond observations (see Table 1), with traces of outburst-like activity in the outer wings of the Balmer lines. Due to this and the limited number of spectra, the data sets cannot be combined to better constrain the period.

To give an impression of the 1s99c and ohp01 data, the He I 4388 line is shown from the ls99c run, while the figure for Si III 4568 was constructed from the ohp01 observations (Fig. 5).

$\pi$ Aqr: The star had a very strong emission spectrum before 1995/96 (Bjorkman et al. 2002). No short-term periodic behavior is known for $\pi$ Aqr, but recently Bjorkman et al. (2002) reported it to be a binary with a period of 84.1 d. Our data, obtained during the low emission phase after 1996 confirms such a timescale in the Balmer emission lines. Three spectra in October 2001 show a relatively strong $\mathrm{H} \alpha$ emission filling up the absorption and a peak height of about 1.1 of the continuum, but by 2002 the emission had weakened again to the previous value, with peak heights of about 0.9 of the ambient continuum.

Multiple, very sharp and rapidly variable absorption spikes are present in all lines normally formed photospherically (Figs. 11 and 12). They were present in every spectrum taken. The width of the absorption spike scales with the expected thermal broadening of the respective element forming the line. No other star in the observed sample exhibits such strong sharp transients. These features might be due to similar processes as the line transients described by Smith (1989) for He I lines of $\lambda$ Eri and also present in HR 4009 (see Fig. 7). But the ordered behavior and strength, especially in metal lines, would still be unique. 

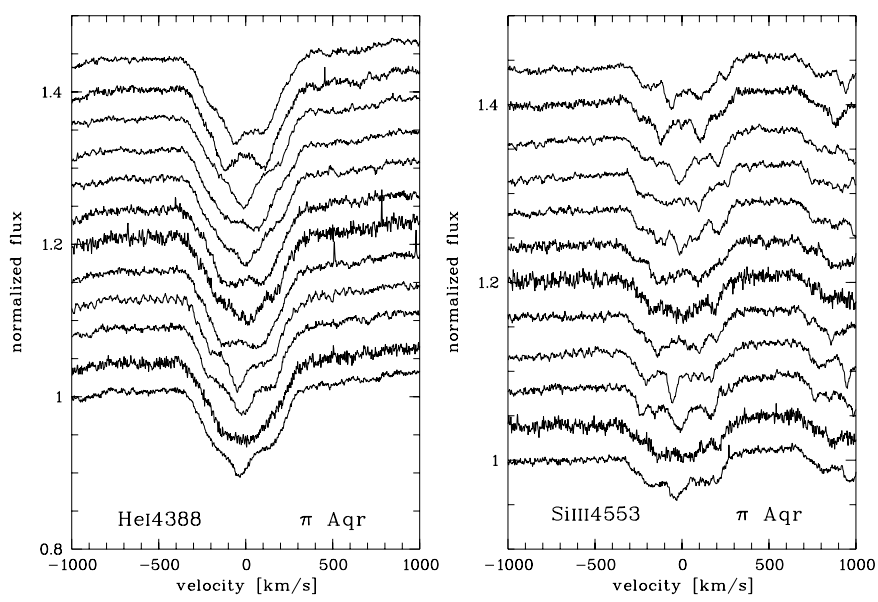

Fig. 11. The $l p v$ of $\pi$ Aqr observed in 1999 with FEROS is clearly of a different type than in most other objects. The individual spectra are separated by a few days, but the evolution of the features is much faster. The behavior of He I 4388 is typical for Balmer and He I lines, while the metal lines behave like Si III 4553. The sharp absorptions can even be seen in lines which are normally too weak to be detected in a Be star of such a $v \sin i$, like in $\mathrm{N}$ II and O II.
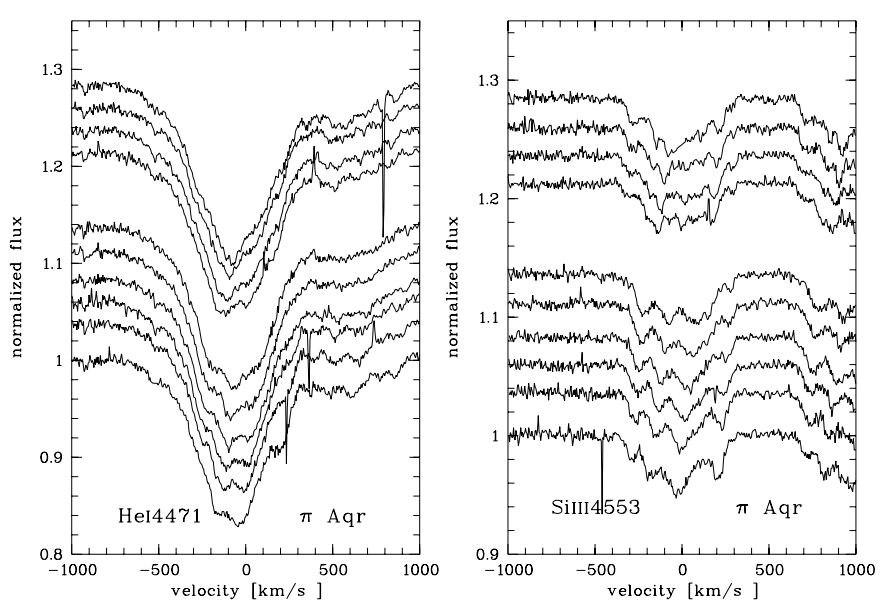

Fig. 12. $\pi$ Aqr observed with AURÉLIE at the OHP $\left(t_{\exp } \approx 10 \mathrm{~min}\right)$. The sharp features clearly propagate across the line profile quickly. The spacing of the spectra is proportional to time (increasing upwards), spanning exactly two hours in total.

o And: Being well known for its photometric variability with highly variable amplitude, this star has the latest spectral subtype in the sample (B6). The dominant photometric period is $1.58 \mathrm{~d}$ (double wave). The main spectroscopic period presented here $(0.694 \mathrm{~d})$ is clearly different from this value and its single-wave equivalent, but also present in the photometric data as third strongest periodicity (Sareyan et al. 1998). Also the amplitude of the spectroscopic variations may vary strongly. In spectroscopic data taken in late 1998 no periodic signature could be found at all (Budovičová et al. 2004). When present, the $l p v$ is different from the one in most other Be stars because the propagation of a feature from the blue to the red side of the line takes longer in phase (about a full cycle) whereas in other Be stars the feature crosses the line width in about 0.6 to 0.7 cycles (Fig. 6).
Other periods tested on the data, in particular the dominant photometric ones, do not sort the spectroscopic $l p v$ at all.

\section{Generalized pulsation model}

Maintz et al. (2003) have modeled the variability of $\omega \mathrm{CMa}$ as $n r p$ in high detail. The stellar parameters of the model by Maintz et al. (2003) are $6 R_{\odot}, 9 M_{\odot}$, a polar temperature of $22000 \mathrm{~K}$, and rotating with $350 \mathrm{~km} \mathrm{~s}^{-1}$ at the equator, which is $80 \%$ of the critical velocity. Depending somewhat on the calibration considered, the fundamental parameters are typical values for stars in the range of B1-2 IV-V, while such a rotational velocity is believed to be normal for a Be star (see e.g. Yudin 2001). The model is pulsating in an $\ell=m=2 g$-mode with a corotating period of $0.92 \mathrm{~d}$ and an amplitude of $35 \mathrm{~km} \mathrm{~s}^{-1}$ as maximal physical velocity on the stellar surface. These parameters reproduce not only the variability, but also the absolute spectrum of $\omega \mathrm{CMa}$ in quite some detail.

Since $\omega \mathrm{CMa}$ is the Be star with the most pronounced $l p v$, however, it is not a priori clear that $\omega \mathrm{CMa}$ is a typical member of its class. It has been mentioned already that differences in $v \sin i$ of individual Be stars are largely due to different inclination $i$, as all Be stars are rapid rotators.

If $\omega \mathrm{CMa}$ were typical and could be observed at higher inclination, its $l p v$ should not differ from the observations of typical high $v \sin i$ Be stars. The differences in the lpvs of low and high $v \sin i$ Be stars, however, are quite pronounced, and the transition between both forms is not explained easily without modeling:

- The variability, concentrated in the wings in low $v \sin i$ stars becomes more evenly spread over the profile width at high $v \sin i$.

- Ramps exceeding $v \sin i$ and spikes become less pronounced and finally undetectable at $v \sin i$ between 150 and $200 \mathrm{~km} \mathrm{~s}^{-1}$ and beyond.

- The variability contrast, generally high and about equal for He I and metal lines in low $v \sin i$ stars decreases as the inclination becomes more equatorial. The variability becomes undetectable with our data in metal lines, but remains visible, though weakly, in He I lines.

The behavior of the pulsational $\omega \mathrm{CMa}$ model at low $v \sin i$, i.e. the formation of spikes and ramps was already discussed by Maintz et al. (2003) and Rivinius et al. (2001a; 2002b). Using the above model parameters for $\omega \mathrm{CMa}$, but changing the inclination at which the model star is observed, four pulsating Be star models were computed for $v \sin i=40,80,160$, and $320 \mathrm{~km} \mathrm{~s}^{-1}$.

The above listed points, which a model should explain, are in fact solved by $n r p$ :

Due to projection effects on the pulsational velocity fields (Maintz et al. 2003; Rivinius et al. 2002b), such $g$-mode pulsation is always seen with about its true physical amplitude, while the maximal rotational velocity is projected with $\sin i$. This immediately explains the decrease of variability contrast with increasing inclination.

Also, in high $v \sin i$ stars the modeled variability exceeds $v \sin i$, but since the excess is equal to the true physical pulsation 

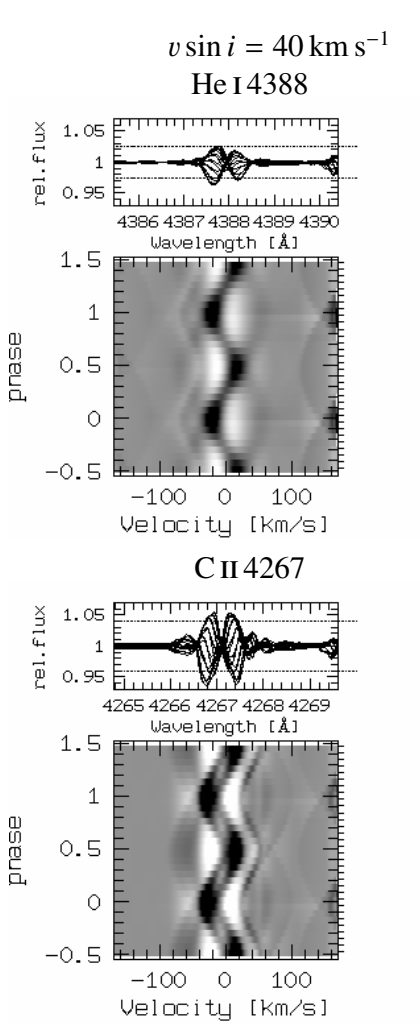

Si III 4553

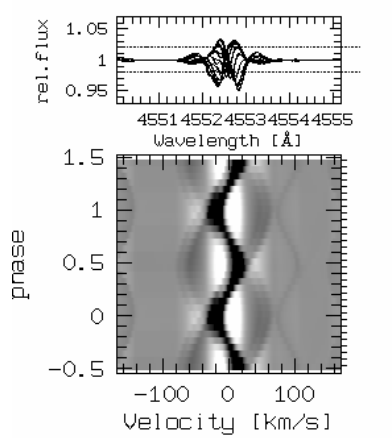

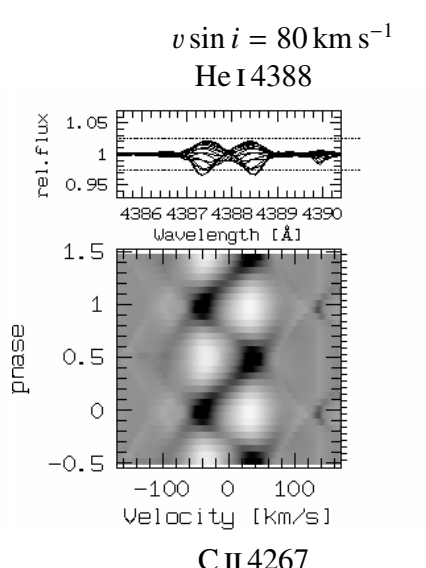

C II 4267

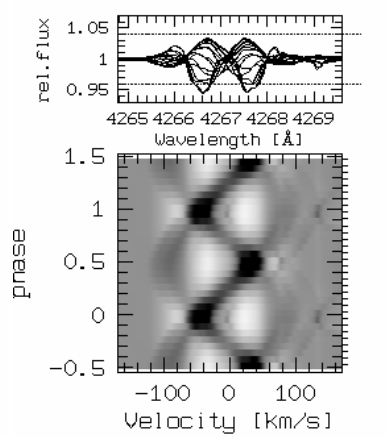

Si III 4553
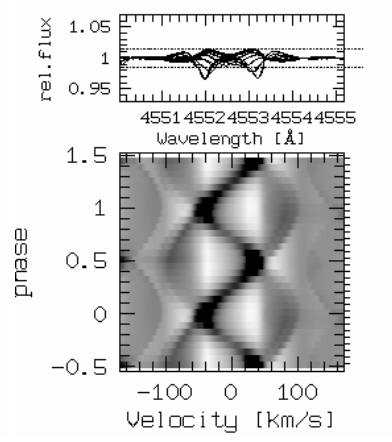
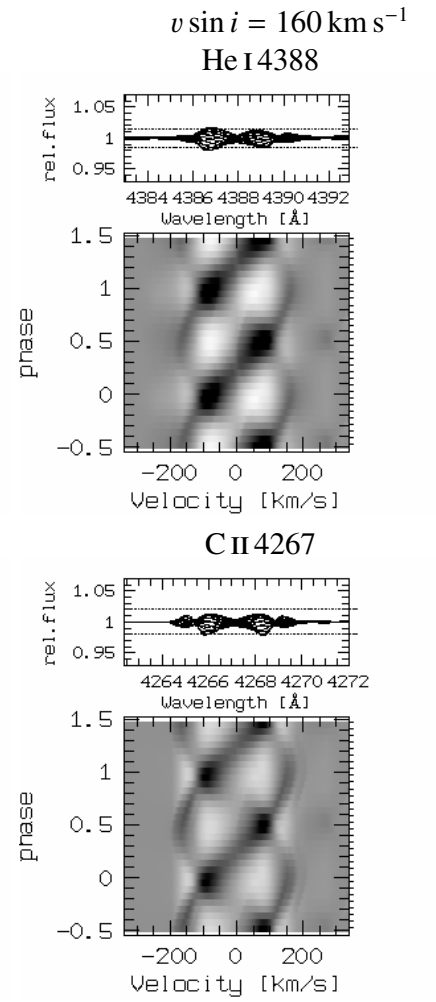

Si III 4553
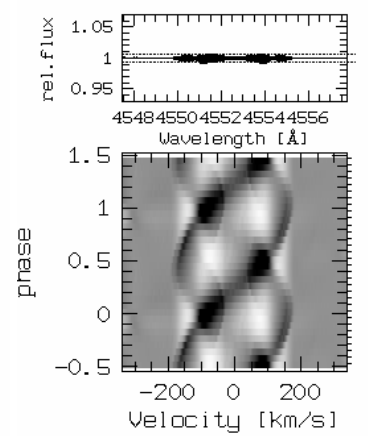
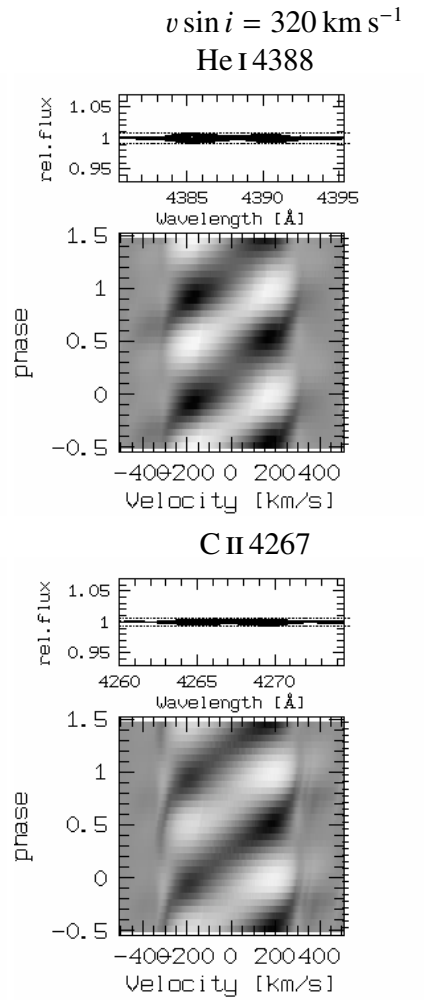

Si III 4553
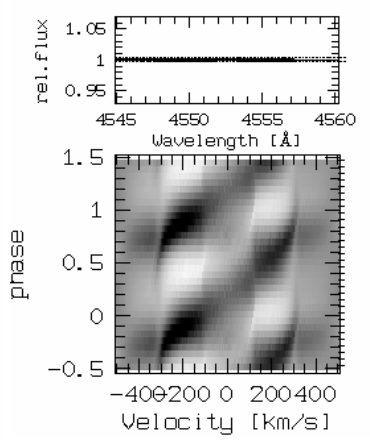

Fig. 13. Phased $l p v$ for $\omega$ CMa-like pulsation, computed for $v \sin i$ values of 40, 80, 160, and $320 \mathrm{~km} \mathrm{~s}^{-1}$. Shown are He I 4388, C II 4267, and S III 4553 The residual variability overplotted on top of each grey-scale picture gives an impression of the decrease of visible contrast with increasing $v \sin i$.

amplitude, this is much less obvious in more equatorial objects, so that ramps should only faintly be seen in observed data with noise contribution, if detectable at all.

The differences between He I and metal lines arise from the different intrinsic line width of the species. For metals, the width is dominated by relatively low thermal broadening, while $\mathrm{He} \mathrm{I}$ and $\mathrm{H}$ I also undergo Stark effects. The smaller this intrinsic line width is, the more prominent spikes are. On the other hand, since the lines with prominent spikes are typically weak ones, these lines are too shallow to be observable in high $v \sin i$ stars.

The $l p v$ of $\mu$ Cen and $\omega$ CMa have been computed quantitatively with physically state-of-the-art line profile and pulsational velocity-field modeling. Due to the individually different stellar parameters, like mass, radius, temperature etc., the comparison with other objects is comparably qualitative and based on the phenomenological appearance of the lpv. Nevertheless, the similarity of Fig. 13 with the observed lpv in Figs. 1 to 4 is striking.

\section{Discussion}

\section{1. $\omega$ CMa like Be stars}

$\omega \mathrm{CMa}$ is compared to other Be in two steps: First, the observed $l p v$ is contrasted to observations of other low $v \sin i$ stars, then the high $v \sin i$ model (see above) is compared to high $v \sin i$ Be stars.

Despite the extraordinary strong lpv of $\omega$ CMa commonalities with other low $v \sin i$ stars, exhibiting weaker $l p v$, can readily be identified (Figs. 1 to 4): Besides FW CMa, HR 4074, and HR 4625 also $\chi$ Oph shows enhanced ramps, though quite weakly, and for $\mu$ Cen a detailed modeling resulted in the same pulsational mode as was derived for $\omega$ CMa (Rivinius et al. 2001a). In all cases, the phase propagation of the variability $\Delta \phi$ 
across the profile is about 0.6 to 0.7 of the full cycle. All the above mentioned early-type Be stars have $v \sin i<160 \mathrm{~km} \mathrm{~s}^{-1}$.

For the two remaining low $v \sin i$ stars in Table 3, 31 Peg and HR 3642, our database is insufficient to investigate the $l p v$ (Fig. 5). However, the spikes present in 31 Peg (Fig. 10) and the published spectra of HR 3642 (Carrier et al. 2002) support the similarity with $\omega \mathrm{CMa}$ also of these stars.

Since these 8 stars represent a significant fraction of all early-type low $v \sin i$ Be stars brighter than 6th magnitude (Slettebak 1982, lists $31 \mathrm{Be}$ stars with $v \sin i<150 \mathrm{~km} \mathrm{~s}^{-1}$, of which 23 are of type earlier than B4), it can be concluded that early-type low $v \sin i$ Be stars in general share a common type of $l p v$. The remaining ones not mentioned here have not been observed in our programme. But there is no reason to assume principally different behavior for those, since most stars were selected on account of their brightness and coordinates.

If $\omega \mathrm{CMa}$ is representative of low $v \sin i$ stars, and the difference between low and high $v \sin i$ is mainly the inclination, $\omega$ CMa should also look like any high $v \sin i$ Be star if it could be observed equator-on. While it is not really feasible to tilt the star, this can easily be tested with the model (Sect. 4).

Comparing Fig. 13 to Figs. 1 to 4 it becomes apparent that also most high $v \sin i$ stars are explained by the model of $\omega \mathrm{CMa}$, tilted to appropriate inclinations (DX Eri, 10 CMa, HR 4009, $\delta$ Cen, $\alpha$ Eri, DU Eri, 66 Oph, PP Car, 28 Cyg, and $\eta$ Cen).

Of the variability of 20 periodic Be stars listed in addition to $\omega \mathrm{CMa}$ in Table 3, six low to intermediate $v \sin i$ stars are directly comparable to $\omega \mathrm{CMa}$, ten intermediate to high $v \sin i$ ones are explainable assuming the same pulsational parameters as for $\omega \mathrm{CMa}$, but different inclination. Two more stars with scarce data, but sufficient to derive a period, are compatible with $n r p$ as well. For two stars, HR 5223 and $\omega$ Ori we could not give a period, but note that the observed $l p v$ does not contradict an interpretation as $\omega$ CMa-like nrp. The three remaining objects with periodic $l p v$ unlike $\omega \mathrm{CMa}$ are discussed in Sect. 5.3.

In addition, the phased $l p v$ presented in the literature also suits the presented model well in many cases. This is not only true for visual comparison of the spectra, like e.g. in HR 3642 (Carrier et al. 2002), or PP Car (Porri \& Stalio 1988). But for stars where mode identifications have been attempted, typically sectorial modes with similar values of $\ell$ and $m$ are derived, as by Neiner et al. (2002) for $\omega$ Ori (see also Table 5).

The long-term coherency of the observed periods also provides an upper limit to potential binary effects. Since no periodic O-C-type variations are found, the Be star must reside close to the center of mass of any presumable binary system (unless all such systems would be pole-on w.r.t. the orbital plane).

\subsection{Asymmetric power distributions}

The power distribution of the $l p v$ can be strongly asymmetric. The appearance of such differences of fifty to several hundred percent between the blue and red sides of the profiles has been discussed in detail by Rivinius et al. (2001a) for $\mu$ Cen. It is

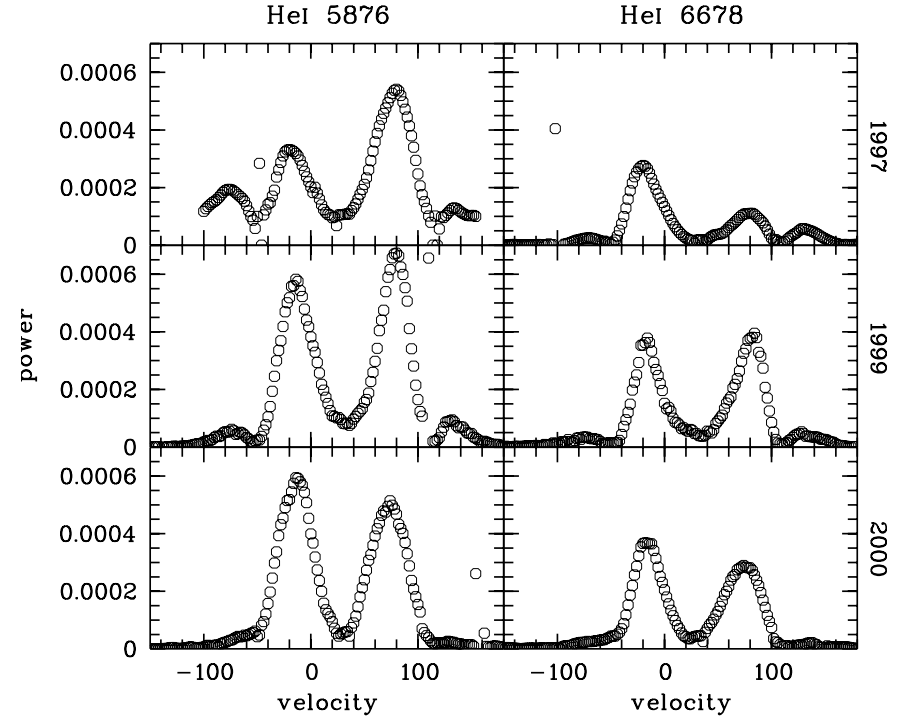

Fig. 14. The power across the spectral line connected to the $l p v$ of $\omega$ CMa. Shown are two lines for three seasons to illustrate the scale this asymmetry can take. Note that the detailed behaviour of both lines seems unrelated, it was also not possible to connect it to the observed outbursts.

also clearly seen in DX Eri and $\delta$ Cen. The cause of the asymmetries is not yet identified. Hypotheses proposed include nonadiabatic effects, wave leakage or other effects of mass-loss related to pulsation.

Weaker power asymmetries of the order of a few percent are much more common. They are seen in most stars, including $\omega$ CMa (Fig. 14, or Štefl et al. 2003b). While for some objects such results might have been artificially introduced by sampling effects, there is no doubt that the majority of those asymmetries are real. Other than for the strong asymmetry discussed above, both red or blue side might be enhanced. In some stars, like $\alpha$ Eri, enhanced variation power is present in the blue wing of some line (e.g. He I 5876) and in the red wing of others, like He I 6678. M. Smith (priv. comm.) proposed NLTE effects of He I line formation to be responsible for this.

In some stars the effect is stronger in lines with typically stronger circumstellar contribution. From season to season the asymmetry may change not only in strength, but even in sign. It is, therefore, not implausible to ascribe such minor asymmetries not to the $n r p$ itself, but to the properties of the emission in the disk regions close to the stars, reacting to the underlying photospheric variability.

\subsection{Objects with discrepant or no photospheric lpv}

The observed $l p v$ of three objects in Table 2 is not explained by an nrp model à la $\omega \mathrm{CMa}$. These are $\kappa \mathrm{CMa}, \lambda$ Eri, and $o$ And, shown in Fig. 6

However, the $l p v$ of these stars, too, generally looks like the one one would expect from nrp. So, it might just require higher values of $\ell$ and $m$ than for $\omega$ CMa to explain the observed behavior in terms of $n r p$. Kambe et al. (2000) in fact propose tesseral $n r p$ modes, i.e. $\ell \neq|m|$, to model the $l p v$ of $\lambda$ Eri, which might also apply to the similar $l p v$ of $\kappa$ CMa. 
For $o$ And the multiperiodicity, observed photometrically in many different seasons, points to $n r p$ as explanation. The lpv of $o$ And differs mainly in phase propagation across the profile from the one of other Be stars, which here is about 1 (meaning the feature is visible over a full cycle, just disappearing redwards when a new one appears on the blue side) as opposed to typically $0.6 \ldots 0.7$ cycles. Some testing with the $n r p$ model code confirms that such a phase propagation would easily be explainable by a sectorial mode, i.e. $\ell=|m|$, with higher $\ell$. In a mode with lower $\ell$, i.e. $\ell=1$, the phase difference should be even less. On the other hand, the strongly variable amplitude was attempted to be explained as an effect of variable magnetic activity, and Sareyan et al. (1998) favor a rotational activity hypothesis together with differential rotation to explain the multiperiodicity.

In any case the $\ell=m=2$ mode explaining the $l p v$ of most other Be stars cannot readily be applied to these three objects.

Two of the investigated stars, $\alpha$ Ara and $\iota$ Ara, did not show any $l p v$, although photometric periods are reported and sufficient spectroscopic data was obtained. If periodic $l p v$ is present in these stars, it must be weaker than in the other objects.

For $\lambda$ Pav the data is too scarce for classification, but $l p v$ is present. The $l p v$ of $\pi$ Aqr, finally, does not fit any of the above descriptions (Figs. 11 and 12).

\subsection{Photospheric multiperiodicity}

For several stars, multiperiodicity was claimed from spectroscopic data. For at least part of these stars, the additional periods are present in the same photosperic lines as the main periods, meaning they arise from the photosphere as well. This is in fact another strong and independent argument to ascribe the $l p v$ to non-radial pulsation.

In one of the best investigated cases, $\mu \mathrm{Cen}$, six photospheric periods are present, grouped around $0.503 \mathrm{~d}$ and $0.28 \mathrm{~d}$ (Rivinius et al. 1998b), and modelled as $n r p$ in modes with $\ell=m=2$ and $\ell=m=3$, respectively (Rivinius et al. 2001a).

Tubbesing et al. (2000) found a similar grouping for the main period of 28 Cyg, using the lsw 97 and ca98 subsets of the data presented here. A preliminary analysis of the additional Ondřejov data confirms the two reported periods at 0.64 and $0.62 \mathrm{~d}$. The main period of $\eta$ Cen could also shown to be double, namely 0.577 and $0.565 \mathrm{~d}$ (see above).

In 66 Oph (Floquet et al. 2002) and $\lambda$ Eri (Kambe et al. $2000)$ shorter periods with larger phase propagation than the main one were reported, but due to unfavorable sampling, $S / N$, and resolving power we could not confirm them in our data. From the published properties, however, these periods seem comparable to the $l p v$ of the short period group in $\mu \mathrm{Cen}$.

\subsection{Secondary circumstellar (transient) periods}

Four stars in the sample exhibit secondary periods within $10 \%$ of their main photospheric ones (Table 4). Taking into account the lines these periods are seen in, namely ones usually formed in the upper photosphere and the close circumstellar environment (e.g. Mg II 4481, He I6678, blue Balmer lines etc., see
Table 4. Secondary (transient) periods of Be stars.

\begin{tabular}{lccc}
\hline \hline Name & HR & $\begin{array}{c}\text { Main } \\
\text { period } \\
{[\mathrm{d}]}\end{array}$ & $\begin{array}{c}\text { Secondary } \\
\text { period } \\
{[\mathrm{d}]}\end{array}$ \\
\hline$\kappa \mathrm{CMa}$ & 2538 & 0.548 & 0.617 \\
$\omega \mathrm{CMa}$ & 2749 & 1.372 & 1.470 \\
$\mu \mathrm{Cen}$ & 5193 & 0.503 & $\approx 0.622$ \\
$\eta$ Cen & 5440 & 0.577 & 0.643 \\
\hline
\end{tabular}

Štefl et al. 2003b), and their typically transient appearance during outbursts only, already Stefl et al. (1998) attributed them to the circumstellar environment. The phase diagrams of the secondary periods are shown in Fig. 15. For $\mu$ Cen only the best sampled event is shown. The patterns look rather similar to each other for non-shell stars, but differs drastically for the shell star $\eta$ Cen. Other than for the photospheric $n r p$ pattern, there seem to be little further aspect effects. This also points to a circumstellar origin of the $l p v$.

The various short-lived periods in $\mu$ Cen support an interpretation as orbital timescale of circumstellar matter ejected shortly before. This is not so easy for the other three objects, where the secondary variability is phase-coherent at least over weeks to months ( $\omega \mathrm{CMa}, \kappa \mathrm{CMa})$, and even years $(\eta \mathrm{Cen})$. Similar behavior was also observed in $\lambda$ Eri by Kambe et al. (1998), who called them intermittent periods.

In any case, contrary to the $n r p$ connected to the primary periods, the secondary periods have to be attributed to processes that strongly interact with or reside in the disk. This would be an additional nrp mode on the stellar surface having an inhomogeneous temperature distribution

- One might assume that the resulting locally variable photospheric radiation influences the line formation properties in the disk immediately above the surface. Such "hot spots" in the inner disk would naturally be phase locked with the pulsation.

- Also, such an (additional) $n r p$ wave pattern could affect the disk by mass loss. Regions above the $n r p$ pattern would have enhanced density. The secondary $l p v$ of $\eta$ Cen might be explained if one assumes such a higher density region to show emission if seen next to the limb of the star, which would be at high projected velocities, and in absorption if in front of the star (Balona 1999).

However, since purely photospheric lines do not show any trace of the secondary periods, such mechanisms are not very likely.

- Alternatively, the secondary periods could be the rotational ones of the stars, which interact with the disk through magnetic processes. But the nrp modeling results for $\omega \mathrm{CMa}$ point to a significantly shorter rotation period of about $1.1 \mathrm{~d}$, compared to the transient period of $1.47 \mathrm{~d}$. On the other hand, Neiner et al. (2003) recently has found a period of $1.2 \mathrm{~d}$ in $\omega$ Ori in addition to the main photopsheric one of about $1.0 \mathrm{~d}$, which seems connected to magnetic field modulation, and thus to be rotational. The period is prominent only in the UV wind, however, and only hardly present in optical lines. 

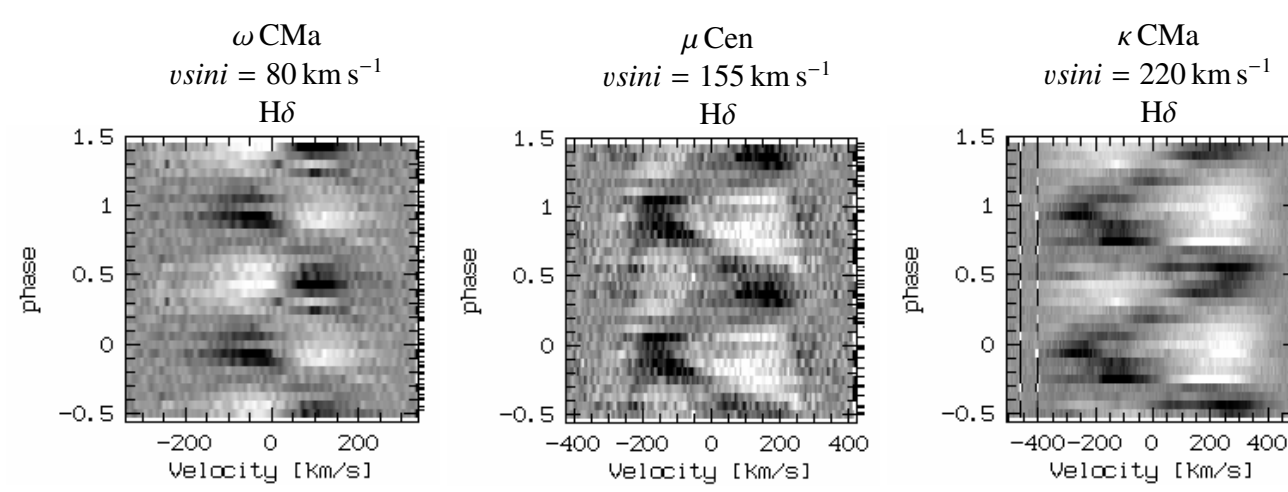

$\mathrm{H} \delta$

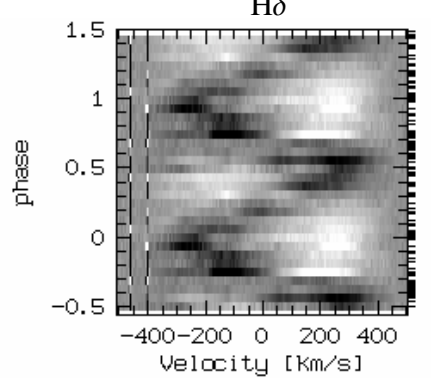

He I 6678
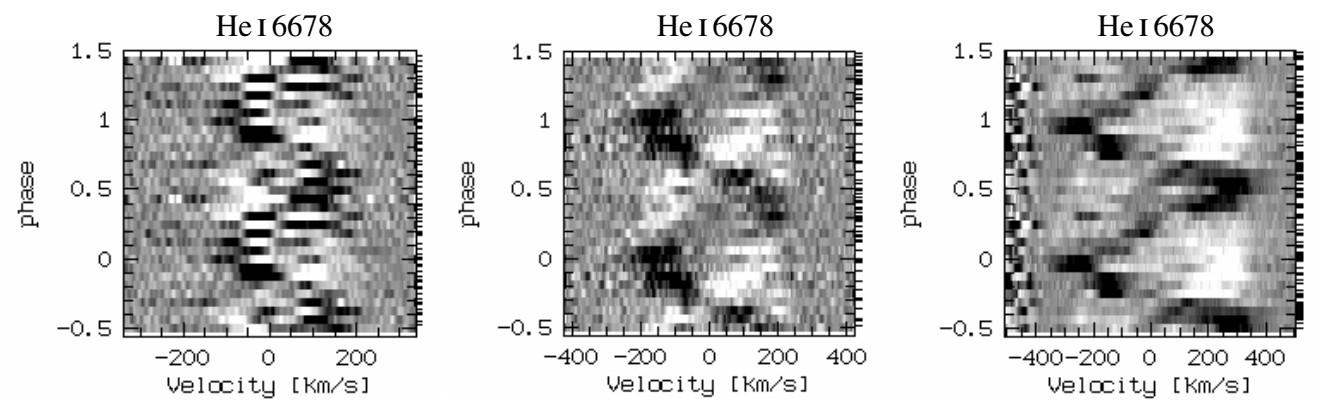

He I 4388
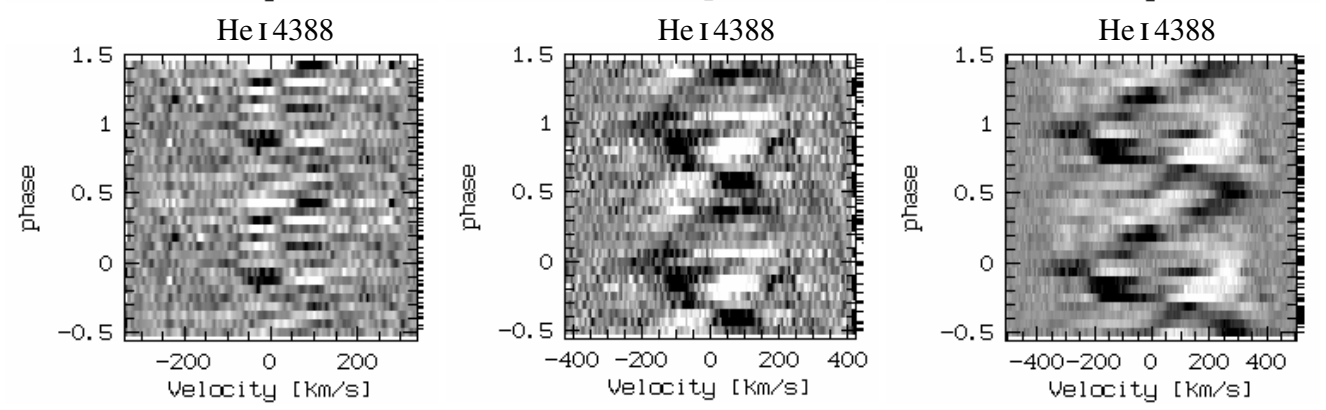

$\eta$ Cen
$v \sin i=350 \mathrm{~km} \mathrm{~s}^{-1}$

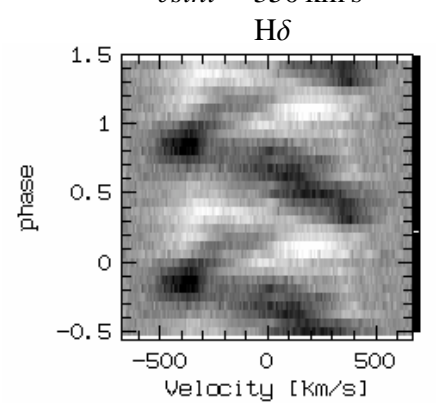

He I 6678

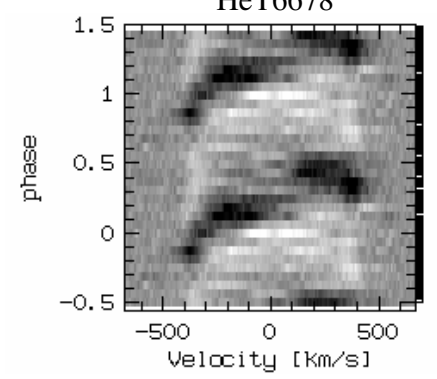

He I 4388

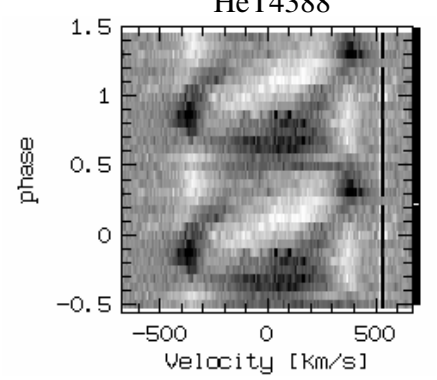

Fig. 15. l $p v$ phased with transient periods of four Be stars, shown for $\mathrm{H} \delta$ and two He I lines. The high scatter in the He I lines of $\omega \mathrm{CMa}$ is due to the main period, that is too strong to be completely averaged out in a data string of 45 spectra obtained during the few weeks of the outburst. The periods are given in Table 4.

- Finally, despite their stability, the secondary periods could entirely be intrinsic to the inner disk, e.g. as the period of a density disturbance. The secondary period of $\eta \mathrm{Cen}$, remaining phase-coherent over several years, is hardly explained by such an interpretation, however.

Although no firm answer can currently be given, the striking differences in the appearance of the secondary $l p v$ from line to line, e.g. in $\eta$ Cen (Fig. 15), can hardly be explained with photospheric line formation properties, but most likely require NLTE effects to be taken into account.

\subsection{Photometric vs. spectroscopic periods}

Most of the objects investigated spectroscopically here were also subject of photometric campaigns. Other than the periodic $l p v$, which is relatively easy to disentangle from circumstellar effects by using different lines, the photometric variations not only include the periodic photospheric, but also the circumstellar variability on all different timescales between hours and decades, convolved into a single number.
Photometrically determined periods, therefore, have to be taken with care, especially since transient secondary periods might additionally be present (Štefl et al. 1999).

Searching the literature for photometric periods of the 21 stars with identified periodic $l p v$ in our sample (Table 3), we found the following:

- In 8 stars, no photometric period was detected. These stars typically have low to intermediate $v \sin i$.

- In 5 stars, the periods agree, and no further period is present. These stars typically have intermediate to high $v \sin i$.

- In 5 stars, the periods do not agree. There is no range in $v \sin i$ clearly preferred in these objects.

- In 3 stars, several periods were detected, including the main spectroscopic one. Since this is not always the one with the largest light amplitude, identification of the main (meaning here: photospheric and long-term coherent) period is only possible if spectroscopic data is taken into account.

On the other hand, there are many later-type Be stars with welldefined photometric periods, which hardly show any variability 
in spectroscopic data. Some of them were also observed within the framework of this project, but even with FEROs data $l p v$ was not found, confirming again the negative result of Baade (1989). o And, B6 III, the latest-type object we detected $l p v$ in, is already an exceptional case.

A spectroscopic study of early-type low $v \sin i$ Be stars in NGC 330 in the SMC gave negative results, i.e. lpv was not found at all, although photometric periods are well established (Baade et al. 2002b) and the observational data should have enabled a detection, if the $l p v$ properties were the same as for Galactic Be stars. This may point to different variability mechanisms of Be stars in the Magellanic Clouds than in our Galaxy, although further data is needed to ascertain these results.

Clearly, photometric studies need to be assisted by spectroscopic observations in order to allow for the unambiguous identification of the photometric variability belonging to the periodic lpv (see also Sect. 5.7 below). Simultaneous observations with both techniques are even required to understand the nature of the additional photometric variability on short timescales.

Only for the objects where the photometric variations connected to the pulsation could be isolated further predictions by the $n r p$ hypothesis can be tested. Other than for the $l p v$, which is Doppler-enhanced at low $v \sin i$, the photometric variability is measured with a broadband filter in the continuum. Therefore, the observable amplitudes should decrease with $v \sin i$ if the physical amplitudes on the stellar surface are comparable for individual objects.

To test this prediction, amplitudes were taken from HIPPARCos data (Percy et al. 2002) for four stars with consistent main photospheric $l p v$ and photometric periods, and from Balona et al. (1987) for $\alpha$ Eri. For three more stars with ambiguous periods, the photometric amplitude for the main one is given by Štefl et al. (1999 $\omega$ CMa), Percy et al. (2002 $\delta$ Cen), and Sareyan et al. (1998 $o$ And). For the 8 stars without any period being detected in photometry, zero amplitude was assumed.

With the exception of three outliers, these stars form a well defined band from zero amplitude at low $v \sin i$ to 0.025 mag at high $v \sin i$ (Fig. 16). The three outliers are PP Car, 66 Oph, and HR 4009, having intermediate $v \sin i$ of about 200 to $250 \mathrm{~km} \mathrm{~s}^{-1}$ but no detected photometric periodicity. These stars are not the most intensively observed ones, however. The spectroscopic $l p v$ is quite weak, partly only recently detected (see Sect. 3). Therefore, the assumption of similar physical amplitudes on the stellar surfaces might not hold for these three stars.

\subsection{The rotational modulation hypothesis}

This hypothesis ascribed the periodic $l p v$ to circumstellar clouds slightly above the photosphere, which supposedly are forced into co-rotation by magnetic effects. The main arguments in favor of this were:

1. The photometric lpv periods and rotational periods deduced from $v \sin i$ and spectral type are statistically identical to within $\sigma=7 \%$ (Balona 1995). Statistical problems with this analysis were already addressed by Baade (1996). In addition, especially for lower $v \sin i$ the determination of

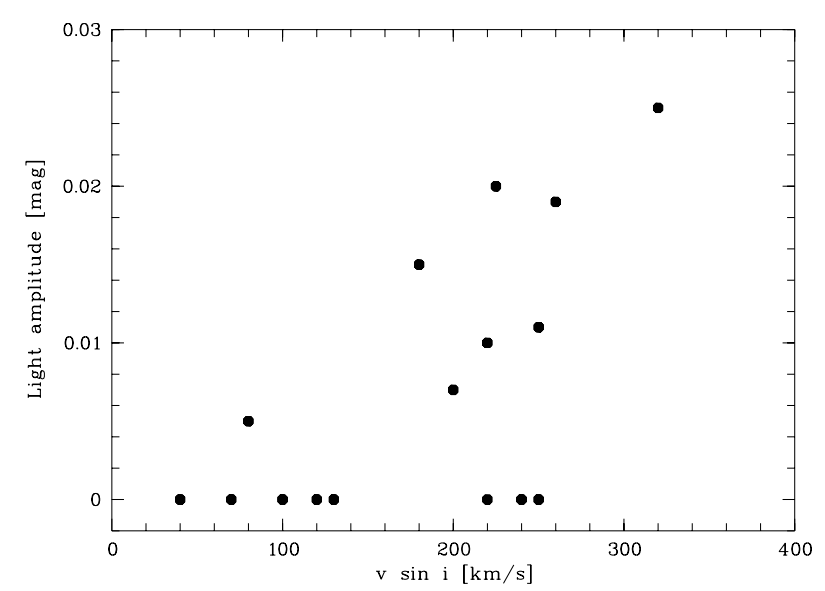

Fig. 16. The photometric amplitude attributed to the main photospheric period, as derived from spectroscopic data, plotted vs. the $v \sin i$ of the star. Since Be stars are rotating rapidly, a low $v \sin i$ is due to pole-on inclination, where $n r p$ induced photometric variations cancel. By contrast to the spectroscopic $l p v$, they are maximal for stars seen equator-on.

the main periods by photometry alone is often ambiguous (see Sect. 5.6 and Table 3). The true rotational velocities and radii of individual Be stars, used to derive the rotational periods, are not well constrained, either.

2. The Balmer lines vary on top of the emission, therefore the variation must be circumstellar. This argument is easily disproved, since the line profiles of non-shell stars are linear superpositions of photospheric and disk contribution. Without cross-checking pure emission and pure absorption profiles such a claim cannot be made. As Štefl et al. (2003b) and Maintz et al. $(2000,2003)$ have shown, the $l p v$ on top of the Balmer lines of $\omega \mathrm{CMa}$ is well explained by photospheric nrp. Similarly, the residual variability patterns are easily misinterpreted as due to physical emission or absorption. Such claims require the undisturbed profiles to be known. However, the traveling bumps due to $n r p$ are neither emission nor absorption, but only a redistribution of the local photospheric equivalent width contributions from the stellar surface in velocity space. Accordingly, the undisturbed profiles can only be derived from detailed modeling.

Independent support against a circumstellar interpretation of the $l p v$ comes from HR 4074, $l p v$-wise almost a twin of $\omega \mathrm{CMa}$, but without any trace of line-emission for at least a century (Štefl et al. 2002), eliminating any potential circumstellar contribution.

3. The complicated appearance of the lpv is not explainable by nrp. We note that of the seven stars investigated in detail to support this stance, three are shell objects ( $\zeta$ Tau, Balona $\&$ Kaye 1999; $\eta$ Cen Balona 1999; and $\epsilon$ Cap Balona \& Lawson 2001) for which a circumstellar contribution of the disk to the absorption lines is not surprising. The example of $\eta$ Cen above illustrates the difficulties of the analysis of such objects. For the other Be stars, ( $\omega \mathrm{CMa}$, Balona et al. 1999; $\omega$ Ori, Balona et al. 2001a; $\mu$ Cen, 
Table 5. Types of $l p v$ observed. Detections which are uncertain are listed in parentheses, periods taken from the literature but not confirmed by our data are marked with $*$ (see Sect. 3 for references). A double $\sqrt{ } \sqrt{ }$ indicates $\mu$ Cen-like closely-spaced multiperiodicity. Where available, mode determinations for the main periods derived by other authors are given in the last three columns.

\begin{tabular}{|c|c|c|c|c|c|c|c|c|c|c|}
\hline \multirow[t]{3}{*}{ Name } & \multirow[t]{3}{*}{ HR } & \multicolumn{9}{|c|}{ Line profile variability type } \\
\hline & & \multirow{2}{*}{$\begin{array}{c}\omega \text { CMa-like } \\
\text { period } \\
\ell=m=+2\end{array}$} & \multirow[t]{2}{*}{$\begin{array}{l}\text { Non- } \omega \mathrm{CMa} \\
\text { period }\end{array}$} & \multirow[t]{2}{*}{ Stable } & \multirow[t]{2}{*}{$\begin{array}{l}\text { Aperiodic } \\
\text { or N/A }\end{array}$} & \multirow[t]{2}{*}{$\begin{array}{l}\text { Additional } \\
\text { short period }\end{array}$} & \multirow[t]{2}{*}{$\begin{array}{l}\text { Secondary } \\
\text { period }\end{array}$} & \multicolumn{3}{|c|}{$\begin{array}{l}\text { Main mode determ. } \\
\text { by other authors }\end{array}$} \\
\hline & & & & & & & & $\ell$ & $m$ & ref. \\
\hline$\alpha$ Eri & 472 & $\sqrt{ }$ & - & - & - & - & - & 2 & -2 & 1 \\
\hline DU Eri & 1423 & $\sqrt{ }$ & - & - & - & - & - & 2 & -2 & 1 \\
\hline DX Eri & 1508 & $\sqrt{ }$ & - & - & - & - & - & & - & \\
\hline$\lambda$ Eri & 1679 & - & $\sqrt{ }$ & - & $*$ & * & - & 2,4 & $-2,-2$ & 1,2 \\
\hline$\omega$ Ori & 1934 & $*,(\sqrt{ })$ & - & - & - & - & - & $2-3$ & \pm 2 & 3 \\
\hline $10 \mathrm{CMa}$ & 2492 & $\sqrt{ }$ & - & - & - & - & - & & - & \\
\hline$\kappa \mathrm{CMa}$ & 2538 & - & $\sqrt{ }$ & - & - & - & $\sqrt{ }$ & & - & \\
\hline$\omega \mathrm{CMa}$ & 2749 & $\sqrt{ }$ & - & - & - & - & $\sqrt{ }$ & 2 & +2 & 4,5 \\
\hline \multirow[t]{4}{*}{ FW CMa } & 2825 & $\sqrt{ }$ & - & - & - & - & - & & - & \\
\hline & 3642 & $(\sqrt{ })$ & - & - & - & - & - & & - & \\
\hline & 4009 & $\sqrt{ }$ & - & - & $\sqrt{ }$ & - & - & & - & \\
\hline & 4074 & $\sqrt{ }$ & - & - & - & - & - & & - & \\
\hline PPCar & 4140 & $\sqrt{ }$ & - & - & - & - & - & & - & \\
\hline \multirow[t]{2}{*}{$\delta$ Cen } & 4621 & $\sqrt{ }$ & - & - & - & - & - & & - & \\
\hline & 4625 & $\sqrt{ }$ & - & - & - & - & - & & - & \\
\hline \multirow[t]{2}{*}{$\mu$ Cen } & 5193 & $\sqrt{ } \sqrt{ }$ & - & - & - & $\sqrt{ }$ & $\sqrt{ }$ & 2 & +2 & 6,7 \\
\hline & 5223 & $(\sqrt{ })$ & - & - & - & - & - & & - & \\
\hline$\eta$ Cen & 5440 & $\sqrt{ } \sqrt{ }$ & - & - & - & - & $\sqrt{ }$ & $2,1-3,3$ & $-2,-,-$ & $1,8,9$ \\
\hline$\chi \mathrm{Oph}$ & 6118 & $\sqrt{ }$ & - & - & - & - & - & & - & \\
\hline$\iota$ Ara & 6451 & - & - & $\sqrt{ }$ & - & - & - & & - & \\
\hline$\alpha$ Ara & 6510 & - & - & $\sqrt{ }$ & - & - & - & & - & \\
\hline $66 \mathrm{Oph}$ & 6712 & $\sqrt{ }$ & - & - & - & * & - & $2-3$ & & 10 \\
\hline$\lambda \mathrm{Pav}$ & 7074 & - & - & - & $\sqrt{ }$ & - & - & & - & \\
\hline $28 \mathrm{Cyg}$ & 7708 & $\sqrt{ } \sqrt{ }$ & - & - & - & - & - & 2 & -2 & 1 \\
\hline $31 \mathrm{Peg}$ & 8520 & $(\sqrt{ })$ & - & - & - & - & - & & - & \\
\hline$\pi$ Aqr & 8539 & - & - & - & $\sqrt{ }$ & - & - & & - & \\
\hline$o$ And & 8762 & - & $\sqrt{ }$ & - & - & - & - & & - & \\
\hline
\end{tabular}

References: 1: Peters \& Gies (2000), 2: Kambe et al. (1998), 3: Neiner et al. (2002), 4: Baade (1982), 5: Maintz et al. (2003), 6: Baade (1984a), 7: Rivinius et al. (2001a), 8: Janot-Pacheco et al. (1999), 9: Levenhagen et al. (2003), 10: Floquet et al. (2002).

Balona et al. 2001b; and $\lambda$ Eri, Balona $\&$ James 2002) we refer to Table 5 and Sect. 3 and the references therein.

Among the objects for which we have enough data for an independent investigation, only the case of $\lambda$ Eri (Balona \& James 2002) clearly withstands an explanation as an $\omega$ CMalike Be star, i.e. as non-radial pulsator in an $\ell=m=2$ mode. This is not a principal problem of the $n r p$ hypothesis, however (Sect. 5.3).

For $\zeta$ Tau and $\epsilon$ Cap, we do not have sufficient data to countercheck the results for these stars in the light of $n r p$, but none of the published results seem to pose principal problems, certainly less than seemingly did $\eta$ Cen.

The dependence of photometric amplitude on inclination (see above, Fig. 16) and the presence of $l p v$ spanning the full range of $\pm v \sin i$ (and more) would require the circumstellar clouds to concentrate above the equator. The prominence of the $l p v$ in nearly pole-on stars, much stronger than in the equatoron ones, is not explainable by equatorial corotating clouds, but requires a velocity field dominating the $l p v$.

\section{Conclusions}

A total of 27 early-type Be stars were investigated in this study, using more than 3000 spectra. Two of the studied objects, $\mu$ Cen and $\omega \mathrm{CMa}$, were already previously investigated in detail (Rivinius et al. 1998b,c, 2001a; Štefl et al. 2003a,b; Maintz et al. 2003).

$\lambda$ Eri is sometimes referred to as prototypical for the $l p v$ of early-type Be stars. However, as the data shows it is in fact a rather unusual case. Line profile variable Be stars should, therefore, not globally be called $\lambda$ Eri-type stars anymore. 
Most of these objects undergo line emission outbursts. In the case of $\mu$ Cen the outburst timing could be attributed to the multiperiodic $l p v$ already by Rivinius et al. (1998a,b). Two more stars with similar multiperiodic properties were identified, 28 Cyg (Tubbesing et al. 2000) and $\eta$ Cen (this study), without being able to establish such a close connection to the outburst timing as in $\mu$ Cen, however. Other objects showing outbursts are equally well observed, but only a single pulsational lpv period is present ( $\omega$ CMa: Štefl et al. 2003b, or $\omega$ Ori: Neiner et al. 2003, for instance).

Some stars show additional periods during outbursts, located in the near circumstellar environment. These secondary, transient periods might even dominate the photometric behavior. It is not clear whether all observed occurrences of transient periods can be explained by a single mechanism, like orbital motion of ejected gas, or some phenomenon phase-locked to the underlying photosphere. Additional, aperiodic short-lived phenomena are present in several stars, but the data presented in this work do not allow to determine their nature.

The analysis and modeling of the periodic line profile variability of $\mu$ Cen and $\omega$ CMa revealed them to be non-radial pulsators with $\ell=m=+2$ (Rivinius et al. 2001a; Maintz et al. 2003). In this work, it was tested whether such $n r p$ can explain other early-type Be stars as well, assuming that the differences in morphology of the $l p v$ arise almost entirely from differences in the individual inclination of the objects. This test was performed by

1. comparing the observed variability of $\omega \mathrm{CMa}$ to that of other low inclination Be stars

2. comparing the $n r p$ model of $\omega \mathrm{CMa}$, tilted to more equatorial viewing angles, to Be stars with higher $v \sin i$.

It turned out that, in addition to $\mu \mathrm{Cen}$ and $\omega \mathrm{CMa}, 14$ more objects show periodic $l p v$ well explainable by $n r p$ with $\ell=m=2$. For four more stars this is likely, but the observational data is not sufficient for a firm conclusion. Three stars exhibit a different type of periodic $l p v$. This does not necessarily exclude $n r p$ for these objects but probably only requires different modes than $\ell=m=2$. Two objects show $l p v$, but the data is insufficient for any conclusion, and finally two stars were stable within the detection limits (Table 5).

Summarizing this work, the low-order lpv seen in the large majority of early-type Be stars is due to non-radial pulsation, typically with $\ell=m=2$.

The relevance of this result for the understanding of the $\mathrm{Be}$ phenomenon still remains to be evaluated; late-type Be stars, being much less photospherically active, indicate either that there is no causal link or that early- and late-type Be stars require different explanations.

Acknowledgements. We are grateful to colleagues and students at the Heidelberg Landessternwarte, the Astronomical Institute in Ondřejov and many more institutes for the enthusiastic participation in the observations. Special thanks go to A. Kaufer, O. Stahl, and B. Wolf for the observations of Be stars during FEROs commissioning and guaranteed time. We also thank M. Dennefeld and the OHP for organizing the 2nd NEON observing school, during which some of the data were obtained. The comments of M. Floquet, the referee, were very constructive and helpful in improving this manuscript.

Financial support was granted by the DFG (Wo 296/20, Sta 288/5, Ap 19/7, 436 TSE 113/18 and 41), the Academy of Sciences and Grant Agency of the Academy of Sciences of the Czech Republic (436 TSE 113/18 and 41, AA3003001), and the LSW Förderverein.

This study made use of the Simbad and ADS databases.

\section{References}

Baade, D. 1982, A\&A, 105, 65

Baade, D. 1984a, A\&A, 135, 101

Baade, D. 1984b, A\&A, 134, 105

Baade, D. 1989, A\&A, 222, 200

Baade, D. 1996, Be Star Newslett., 31, 7

Baade, D., Rivinius, Th., \& Štefl, S. 2002a, ESO Messenger, 107, 24

Baade, D., Rivinius, Th., Štefl, S., \& Kaufer, A. 2002b, A\&A, 383, L31

Balona, L. A. 1990, MNRAS, 245, 92

Balona, L. A. 1995, MNRAS, 277, 1547

Balona, L. A. 1999, MNRAS, 306, 407

Balona, L. A., \& James, D. J. 2002, MNRAS, 332, 714

Balona, L. A., \& Kaye, A. B. 1999, ApJ, 521, 407

Balona, L. A., \& Lawson, W. A. 2001, MNRAS, 321, 131

Balona, L. A., Engelbrecht, C. A., \& Marang, F. 1987, MNRAS, 227, 123

Balona, L. A., Cuypers, J., \& Marang, F. 1992, A\&AS, 92, 533

Balona, L. A., Aerts, C., \& Štefl, S. 1999, MNRAS, 305, 519

Balona, L. A., Aerts, C., Božić, H., et al. 2001a, MNRAS, 327, 1288

Balona, L. A., James, D. J., Lawson, W. A., \& Shobbrook, R. R. 2001b, MNRAS, 324, 1041

Bjorkman, K. S., Miroshnichenko, A. S., McDavid, D., \& Pogrosheva, T. M. 2002, ApJ, 573, 812

Briquet, M., Aerts, C., \& De Cat, P. 2001, A\&A, 366, 121

Budovičová, A. et al. 2004, A\&A, in prep.

Carrier, F., Burki, G., \& Burnet, M. 2002, A\&A, 385, 488

Cuypers, J., Balona, L. A., \& Marang, F. 1989, A\&AS, 81, 151

Dachs, J., \& Lemmer, U. 1991, in Rapid Variability of OB-Stars: Nature and Diagnostic Value, ed. D. Baade, ESO Conf. and Workshop Proc. No. 36 (Garching: ESO), 103

de Cat, P. 2002, in Radial and Nonradial Puslation as Probes of Stellar Physics, ed. C. Aerts, T. R. Bedding, \& J. Christensen-Dalsgaard, ASP Conf. Ser. 259: IAU Colloq., 185, 196

Domiciano de Souza, A., Kervella, P., Jankov, S., et al. 2003, A\&A, 408, L47

Floquet, M., Neiner, C., Janot-Pacheco, E., et al. 2002, A\&A, 394, 137

Hahula, M. E., \& Gies, D. 1994, in Pulsation, Rotation, and Mass Loss in Early-type Stars, ed. L. A. Balona, H. F. Henrichs, \& J. M. Le Contel (Dordrecht: Kluwer), IAU Symp., 162, 100

Hanuschik, R. W., Dachs, J., Baudzus, M., \& Thimm, G. 1993, A\&A, 274,356

Hanuschik, R. W., Hummel, W., Sutorius, E., Dietle, O., \& Thimm, G. 1996, A\&AS, 116, 309

Hubert, A. M., \& Floquet, M. 1998, A\&A, 335, 565

Janot-Pacheco, E., Jankov, S., Leister, N. V., Hubert, A. M., \& Floquet, M. 1999, A\&AS, 137, 407

Kambe, E., Ando, H., Hirata, R., et al. 1993, PASP, 105, 1222

Kambe, E., Fabregat, J., \& Hirata, R. 1998, in A Half Century of Stellar Pulsation Interpretations, ed. P. A. Bradley, \& J. A. Guzik, ASP Conf. Ser., 135, 145 
Kambe, E., Hirata, R., \& Fabregat, J. 2000, in The Be Phenomenon in Early Type Stars, ed. M. A. Smith, H. F. Henrichs, \& J. Fabregat, ASP Conf. Ser. 214: IAU Colloq., 175, 252

Kaufer, A. 1998, in Rev. in Mod. Astron. No. 11, ed. E. Schielicke, 177

Kaufer, A., Stahl, O., Tubbesing, S., et al. 1999, ESO Messenger, 95, 8

Kaufer, A., Stahl, O., Wolf, B., et al. 1996, A\&A, 305, 887

Kaufer, A., Wolf, B., Andersen, J., \& Pasquini, L. 1997, ESO Messenger, 89, 1

Levenhagen, R. S., Leister, N. V., Zorec, J., et al. 2003, A\&A, 400, 599

Maintz, M., Rivinius, Th., Tubbesing, S., et al. 2000, in The Be Phenomenon in Early Type Stars, ed. M. A. Smith, H. F. Henrichs, \& J. Fabregat, ASP Conf. Ser. 214: IAU Colloq., 175, 244

Maintz, M., Rivinius, Th., Štefl, S., et al. 2003, A\&A, 411, 181

Neiner, C., Hubert, A.-M., Floquet, M., et al. 2002, A\&A, 388, 899

Neiner, C., Hubert, A. M., Frémat, Y., et al. 2003, A\&A, 409, 275

Pavlovski, K., Harmanec, P., Božić, H., et al. 1997, A\&AS, 125, 75

Percy, J. R., Hosick, J., Kincaide, H., \& Pang, C. 2002, PASP, 114, 551

Peters, G. J., \& Gies, D. R. 2000, in The Be Phenomenon in Early Type Stars, ed. M., A. Smith, H. F. Henrichs, \& J. Fabregat, ASP Conf. Ser. 214: IAU Colloq., 175, 375

Peters, G. J., \& Penrod, G. D. 1988, in A Decade of UV Astronomy with the IUE Satellite, 2, 117

Porri, A., \& Stalio, R. 1988, A\&AS, 75, 371

Reiners, A., Stahl, O., Wolf, B., Kaufer, A., \& Rivinius, Th. 2000, A\&A, 363, 585

Rivinius, Th. 1999, in Variable and Non-spherical Stellar Winds in Luminous Hot Stars, ed. B. Wolf, O. Stahl, \& A. W. Fullerton, Lecture Notes in Physics, 523: IAU Colloq., 169, 36

Rivinius, Th., Baade, D., Štefl, S., et al. 1998a, in Cyclical Variability in Stellar Winds, ed. L. Kaper, \& A. Fullerton, ESO Astroph. Symp., 207

Rivinius, Th., Baade, D., Štefl, S., et al. 1998b, A\&A, 336, 177

Rivinius, Th., Baade, D., Štefl, S., et al. 1998c, A\&A, 333, 125

Rivinius, Th., Baade, D., Štefl, S., et al. 2001a, A\&A, 369, 1058

Rivinius, Th., Štefl, S., Stahl, O., et al. 2001b, J. Astron. Data, 7, 5
Rivinius, Th., Baade, D., Štefl, S., \& Maintz, M. 2002a, in Radial and Nonradial Puslation as Probes of Stellar Physics, ed. C. Aerts, T. R. Bedding, \& J. Christensen-Dalsgaard, ASP Conf. Ser. 259: IAU Colloq. 185, 240

Rivinius, Th., Baade, D., Štefl, S., Maintz, M., \& Townsend, R. 2002b, ESO Messenger, 108, 20

Sareyan, J. P., Gonzalez-Bedolla, S., Guerrero, G., et al. 1998, A\&A, 332, 155

Scargle, J. D. 1982, ApJ, 263, 835

Slettebak, A. 1982, ApJS, 50, 55

Smith, M. A. 1989, ApJS, 71, 357

Smith, M. A. 2001, ApJ, 562, 998

Spear, G. G., Mills, J., \& Snedden, S. A. 1981, PASP, 93, 460

Štefl, S., \& Balona, L. A. 1996, A\&A, 309, 787

Štefl, S., Baade, D., Harmanec, P., \& Balona, L. A. 1995, A\&A, 294, 135

Štefl, S., Baade, D., Rivinius, Th., et al. 1998, in A Half Century of Stellar Pulsation Interpretations, ed. P. A. Bradley, \& J. A. Guzik, ASP Conf. Ser., 135, 348

Štefl, S., Aerts, C., \& Balona, L. A. 1999, MNRAS, 305, 505

Štefl, S., Rivinius, Th., \& Baade, D. 2002, in Radial and Nonradial Puslation as Probes of Stellar Physics, ed. C. Aerts, T. R. Bedding, \& J. Christensen-Dalsgaard, ASP Conf. Ser. 259: IAU Colloq., 185, 248

Štefl, S., Baade, D., Rivinius, Th., et al. 2003a, A\&A, 402, 253

Štefl, S., Baade, D., Rivinius, Th., et al. 2003b, A\&A, 411, 167

Štefl, S., Hadrava, P., Baade, D., et al. 2003c, in Stellar Rotation, ed. A. Maeder, \& P. Eenens, IAU Symp., 215, in press

Townsend, R. H. D. 1997, MNRAS, 284, 839

Tubbesing, S., Rivinius, Th., Wolf, B., \& Kaufer, A. 2000, in The Be Phenomenon in Early Type Stars, ed. M. A. Smith, H. F. Henrichs, \& J. Fabregat, ASP Conf. Ser. 214: IAU Colloq., 175, 232

Waelkens, C., Aerts, C., Kestens, E., Grenon, M., \& Eyer, L. 1998, A\&A, 330, 215

Yudin, R. V. 2001, A\&A, 368, 912

Zorec, J., Israelian, G., Ballereau, D., \& Chauville, J. 1996, A\&A, 308,852 